Claremont Colleges

Scholarship@ Claremont

All HMC Faculty Publications and Research

HMC Faculty Scholarship

10-1-1994

\title{
Boundary Conditions for the Diffusion Equation in Radiative Transfer
}

\author{
Richard C. Haskell \\ Harvey Mudd College \\ Lars O. Svaasand \\ University of Trondheim \\ Tsong-Tseh Tsay \\ University of California - Irvine \\ Ti-Chen Feng \\ Harvey Mudd College \\ Matthew S. McAdams \\ Harvey Mudd College \\ See next page for additional authors
}

\section{Recommended Citation}

Haskell, RC, Svaasand, LO, Tsay, TT, Feng, TC, McAdams, MS, Tromberg, BJ. Boundary conditions for the diffusion equation in radiative transfer. J Opt Soc Am A. 1994;11(10): 2727-2741.

This Article is brought to you for free and open access by the HMC Faculty Scholarship at Scholarship @ Claremont. It has been accepted for inclusion in All HMC Faculty Publications and Research by an authorized administrator of Scholarship @ Claremont. For more information, please contact scholarship@cuc.claremont.edu. 
Authors

Richard C. Haskell, Lars O. Svaasand, Tsong-Tseh Tsay, Ti-Chen Feng, Matthew S. McAdams, and Bruce J.

Tromberg 


\title{
Boundary conditions for the diffusion equation in radiative transfer
}

\author{
Richard C. Haskell \\ Harvey Mudd College, Claremont, California 91711 \\ Lars O. Svaasand \\ University of Trondheim, 7000 Trondheim, Norway \\ Tsong-Tseh Tsay
}

Beckman Laser Institute and Medical Clinic, University of California, Irvine, Irvine, California 92715

Ti-Chen Feng and Matthew S. McAdams

Harvey Mudd College, Claremont, California 91711

Bruce J. Tromberg

Beckman Laser Institute and Medical Clinic, University of California, Irvine, Irvine, California 92715

Received January 14, 1994; revised manuscript received April 22, 1994; accepted May 18, 1994

\begin{abstract}
Using the method of images, we examine the three boundary conditions commonly applied to the surface of a semi-infinite turbid medium. We find that the image-charge configurations of the partial-current and extrapolated-boundary conditions have the same dipole and quadrupole moments and that the two corresponding solutions to the diffusion equation are approximately equal. In the application of diffusion theory to frequency-domain photon-migration (FDPM) data, these two approaches yield values for the scattering and absorption coefficients that are equal to within $3 \%$. Moreover, the two boundary conditions can be combined to yield a remarkably simple, accurate, and computationally fast method for extracting values for optical parameters from FDPM data. FDPM data were taken both at the surface and deep inside tissue phantoms, and the difference in data between the two geometries is striking. If one analyzes the surface data without accounting for the boundary, values deduced for the optical coefficients are in error by $50 \%$ or more. As expected, when aluminum foil was placed on the surface of a tissue phantom, phase and modulation data were closer to the results for an infinite-medium geometry. Raising the reflectivity of a tissue surface can, in principle, eliminate the effect of the boundary. However, we find that phase and modulation data are highly sensitive to the reflectivity in the range of $80-100 \%$, and a minimum value of $98 \%$ is needed to mimic an infinite-medium geometry reliably. We conclude that noninvasive measurements of optically thick tissue require a rigorous treatment of the tissue boundary, and we suggest a unified partial-current-extrapolated boundary approach.
\end{abstract}

\section{INTRODUCTION}

During the past five years the application of diffusion theory to radiative transfer has become increasingly more fruitful, particularly in laser diagnostics of biological tissue. ${ }^{1}$ Photon-migration techniques based on diffusion theory have been used to monitor optical properties that reflect the physiological state of tissue. ${ }^{2}$ Photon-density waves, strongly damped wave solutions to the diffusion equation, have been used to detect objects embedded in tissue phantoms. ${ }^{3,4}$ It is important that these laser techniques be noninvasive if they are to be clinically useful, so the optical fibers transporting the laser light must be placed on the surface of the tissue. The presence of a tissue boundary is therefore inevitable, and diffusion theory must account for this boundary if errors of $50 \%$ or more are to be avoided in the measurement of optical properties.

0740-3232/94/102727-15\$06.00
The first attempts to apply an appropriate boundary condition to the diffusion equation in radiative transfer led to awkward results. ${ }^{5}$ The solutions violated the diffusion approximation near the surface, invalidating the derivation of the diffusion equation from the linearized Boltzmann transport equation. However, when Fresnel reflections at the surface are included in the boundary condition, the pressure on the diffusion approximation is somewhat relieved. Two different boundary conditions can be modified to account for Fresnel reflections that arise from the refractive-index mismatch at the tissue-air interface: (1) the extrapolated boundary condition ${ }^{6-9}$ and (2) the partial-current (or radiation) boundary condition. ${ }^{10-12}$

In Section 2 we use the method of images to derive solutions to the diffusion equation with the partial-current and extrapolated boundary conditions. We discover that the image-charge configurations for the partial-current 
and extrapolated boundary conditions have the same dipole and quadrupole moments. As a result, these two solutions to the diffusion equation are nearly the same. Indeed, the extrapolated boundary solution obeys the partial-current boundary condition to a good approximation. This fact motivates a remarkable simplification in the analytical expressions for the phase and modulation of photon-density waves in tissue. These simplified expressions [Eqs. (2.7) below] represent a unification of the partial-current and extrapolated boundary conditions, and they provide an accurate and computationally fast means of accounting for a boundary in noninvasive measurements that are based on diffusion theory.

In Section 3 we present frequency-domain photonmigration (FDPM) data to test and illustrate the predictions of diffusion theory. We find that either the partial-current or the extrapolated boundary approach or the unified partial-current-extrapolated boundary approach can successfully account for the presence of a boundary. We also observe a reduction in the effect of the boundary on phase and modulation data when aluminum foil is placed on the surface. Increasing the reflectivity of the boundary raises the light level near the boundary (and hence the detected signal), renders the diffusion approximation more applicable, and yields FDPM data closer to the data of an infinite-medium geometry. However, for the effect of the boundary to be eliminated and the situation to be converted to an (effective) infinite-medium geometry, the reflectivity of the surface would have to reach an impractically high value (98\%). Hence the only feasible method for probing tissue noninvasively is to account for the boundary with an appropriate boundary condition.

\section{DIFFUSION THEORY IN RADIATIVE TRANSFER}

At least three boundary conditions have been applied to the diffusion equation in radiative transfer. In this section we describe all three and compare their predictions for phase and modulation in FDPM measurements. We begin with a brief derivation of the diffusion equation, starting from the linearized Boltzmann transport equation, and emphasize the approximations and limitations that one must keep in mind while using diffusion theory. We then discuss photon-density waves in an infinite medium and check the validity of the diffusion approximation. Extending our discussion to a half-space geometry, we find that Fresnel reflections at the boundary of a semi-infinite medium save the diffusion approximation from flagrant violation. We note that the partial-current boundary condition specifies the anisotropy in the radiance at the surface, making it easy to check on the validity of the diffusion approximation. We also examine the zero boundary and extrapolated boundary conditions and conclude the section by recommending an approximate form of the partial-current condition, which is actually a simplified version of the extrapolated-boundary condition.

\section{A. Derivation of the Diffusion Equation}

The propagation of electromagnetic waves in scattering media can be described with the Boltzmann transport

$$
\begin{aligned}
& \text { equation }^{13-16} \\
& \begin{array}{l}
\frac{1}{c} \frac{\partial L(\mathbf{r}, \hat{s}, t)}{\partial t}+\nabla \cdot L(\mathbf{r}, \hat{s}, t) \hat{s}=-(\sigma+\beta) L(\mathbf{r}, \hat{s}, t) \\
\quad+\sigma \iint_{4 \pi} L\left(\mathbf{r}, \hat{s}^{\prime}, t\right) f\left(\hat{s} \cdot \hat{s}^{\prime}\right) \mathrm{d} \Omega^{\prime}+Q(\mathbf{r}, \hat{s}, t),
\end{array}
\end{aligned}
$$

where the radiance $L(\mathbf{r}, \hat{s}, t)$ has units $\mathrm{W} /\left(\mathrm{m}^{2} \mathrm{sr}\right)$ and where $\hat{s}$ is a unit vector pointing in the direction of interest. The linear scattering and absorption coefficients, $\sigma$ and $\beta$, are the inverses of the mean free paths for scattering and absorption, respectively, and the normalized differential scattering cross section $f\left(\hat{s} \cdot \hat{s}^{\prime}\right)$ satisfies

$$
\iint_{4 \pi} f\left(\hat{s} \cdot \hat{s}^{\prime}\right) \mathrm{d} \Omega^{\prime}=1 .
$$

The source term $Q(\mathbf{r}, \hat{s}, t)$ represents power injected into a unit solid angle centered on $\hat{s}$ in a unit volume at $\mathbf{r}$. Equation (2.1.2) treats photons as billiard balls undergoing elastic collisions and traveling through the medium at speed $c=\left(3 \times 10^{8} \mathrm{~m} / \mathrm{s}\right) / n$, where $n$ is the refractive index of the medium (typically $n=1.40$ for biological tissues ${ }^{17}$ ). Interference effects of photons are assumed to average to zero. In essence, Eq. (2.1.1) provides a mathematical accounting of incoherent photons.

The similarity of transport equation (2.1.1) to a continuity equation is emphasized by integration over all solid angles and use of the definitions of the fluence rate $\phi$ and the flux $\mathbf{j}$ :

$$
\frac{1}{c} \frac{\partial \phi(\mathbf{r}, t)}{\partial t}+\nabla \cdot \mathbf{j}(\mathbf{r}, t)=-\beta \phi(\mathbf{r}, t)+S(\mathbf{r}, t),
$$

where

$$
\begin{aligned}
S(\mathbf{r}, t) & \equiv \iint_{4 \pi} Q(\mathbf{r}, \hat{s}, t) \mathrm{d} \Omega, \\
\phi(\mathbf{r}, t) & \equiv \iint_{4 \pi} L(\mathbf{r}, \hat{s}, t) \mathrm{d} \Omega, \\
\mathbf{j}(\mathbf{r}, t) & \equiv \iint_{4 \pi} L(\mathbf{r}, \hat{s}, t) \hat{s} \mathrm{~d} \Omega .
\end{aligned}
$$

When scattering is much stronger than absorption $\left(\sigma_{\mathrm{tr}} \gg \beta\right)$, the radiance can be expressed as an isotropic fluence rate $\phi$ plus a small directional flux $\mathbf{j}$, and transport equation (2.1.1) reduces to a diffusion equation. ${ }^{5,13-16}$ We first write the radiance as

$$
L(\mathbf{r}, \hat{s}, t)=\frac{1}{4 \pi} \phi(\mathbf{r}, t)+\frac{3}{4 \pi} \mathbf{j}(\mathbf{r}, t) \cdot \hat{s} .
$$

Substituting this diffusion approximation into Eq. (2.1.1) and then multiplying by $\hat{s}$ and integrating over all solid angles yields

$$
\frac{1}{c} \frac{\partial \mathbf{j}(\mathbf{r}, t)}{\partial t}=-\frac{1}{3} \nabla \phi(\mathbf{r}, t)-\frac{1}{3 D} \mathbf{j}(\mathbf{r}, t),
$$

where

$$
D \equiv \frac{1}{3[(1-g) \sigma+\beta]} \equiv \frac{1}{3 \sigma_{\mathrm{tr}}} \equiv \frac{l_{\mathrm{tr}}}{3},
$$

where $D$ is the photon-diffusion coefficient, $g$ is the average cosine of the scattering angle, $\sigma_{\mathrm{tr}}=(1-g) \sigma+$ $\beta$ is the linear transport coefficient, and $l_{\mathrm{tr}}=1 / \sigma_{\mathrm{tr}}$ is 
the transport mean free path. We have made the additional assumption that the source term $Q$ in Eq. (2.1.1) is isotropic. In steady state, Eq. (2.1.5) yields an expression for the flux analogous to Fick's law:

$$
\mathbf{j}(\mathbf{r}, t)=-D \nabla \phi(\mathbf{r}, t)
$$

Even when the source varies in time, Eq. (2.1.6) is a good approximation for biological tissues if the source frequencies are less than $\sim 1 \mathrm{GHz}$.

The two relations between the fluence rate and the flux can be combined to yield a differential equation in the fluence rate alone. Using Eq. (2.1.3) with the divergence of Eq. (2.1.5) gives

$$
\begin{array}{r}
D \nabla^{2} \phi(\mathbf{r}, t)-\beta \phi(\mathbf{r}, t)=(1+3 D \beta) \frac{1}{c} \frac{\partial \phi(\mathbf{r}, t)}{\partial t}-S(\mathbf{r}, t) \\
+\frac{3 D}{c^{2}} \frac{\partial^{2} \phi(\mathbf{r}, t)}{\partial t^{2}}-\frac{3 D}{c} \frac{\partial S(\mathbf{r}, t)}{\partial t} \cdot
\end{array}
$$

For most biological tissues the scattering and absorption coefficients are in the range $10 / \mathrm{cm}<\sigma_{\mathrm{tr}}<50 / \mathrm{cm}$ and $0.03 / \mathrm{cm}<\beta<0.15 / \mathrm{cm}$ [in vivo, and with $\lambda \cong 650 \mathrm{~nm}$ (Refs. 18 and 19)], so $3 D \beta \ll 1$. If the source varies in time with frequencies of less than $\sim 1 \mathrm{GHz}$, then the last two terms in Eq. (2.1.7) can also be neglected, ${ }^{20}$ leaving the diffusion equation

$$
D \nabla^{2} \phi(\mathbf{r}, t)-\beta \phi(\mathbf{r}, t)=\frac{1}{c} \frac{\partial \phi(\mathbf{r}, t)}{\partial t}-S(\mathbf{r}, t) .
$$

We are interested in solutions to Eq. (2.1.8) for an infinite medium and for a semi-infinite medium with a planar boundary. In the latter case we will focus on the appropriate boundary conditions that must supplement diffusion equation (2.1.8).

B. Infinite-Medium Solutions to the Diffusion Equation In an infinite medium we require only that the fluence rate $\phi$ become small at large distances from the source. The Green's function solution to diffusion equation (2.1.8) for a source pulse of unit energy emitted from the origin at time $t=t^{\prime}$ is $^{21}$

$$
\begin{aligned}
\phi_{G}\left(\mathbf{r}, t-t^{\prime}\right) & =\frac{c}{\left[4 \pi D c\left(t-t^{\prime}\right)\right]^{3 / 2}} \\
& \times \exp \left[-\frac{r^{2}}{4 D c\left(t-t^{\prime}\right)}-\beta c\left(t-t^{\prime}\right)\right]
\end{aligned}
$$

It follows that the fluence rate solution for a harmonic source at the origin emitting power $P \exp (i \omega t)$ is given by

$$
\begin{aligned}
\phi_{\omega}(\mathbf{r}, t) & =\int_{-\infty}^{t} \phi_{G}\left(\mathbf{r}, t-t^{\prime}\right) P \exp \left(i \omega t^{\prime}\right) \mathrm{d} t^{\prime} \\
& =\frac{P \exp (i \omega t)}{4 \pi D} \frac{\exp (-k r)}{r} \\
& =\frac{P}{4 \pi D} \exp \left(-k_{\text {real }} r\right) \frac{\exp \left[-i\left(k_{\text {imag }} r-\omega t\right)\right]}{r},
\end{aligned}
$$

where

$$
k=k_{\text {real }}+i k_{\text {imag }}=\sqrt{\frac{\beta c+i \omega}{D c}}, \quad \tau \equiv \frac{1}{\beta c},
$$

so that

$$
\begin{aligned}
& k_{\text {real }}=\sqrt{\frac{3}{2} \beta \sigma_{\operatorname{tr}}}\left[\sqrt{1+(\omega \tau)^{2}}+1\right]^{1 / 2}, \\
& k_{\text {imag }}=\sqrt{\frac{3}{2} \beta \sigma_{\operatorname{tr}}}\left[\sqrt{1+(\omega \tau)^{2}}-1\right]^{1 / 2} .
\end{aligned}
$$

We performed the integral in Eq. (2.2.2) by changing variables and making use of the Laplace transform identity listed as Eq. (6) in App. V of Ref. 22. The absorption relaxation time, $\tau$, defined in Eq. (2.2.3), ranges typically from 0.3 to $1.5 \mathrm{~ns}$ in biological tissue. The fluence rate solution in Eq. (2.2.2) has the form of a spherical wave, often called a diffuse photon-density wave, and is strongly overdamped even in the absence of absorption. Photondensity waves can be characterized by their modulation wavelength, $\lambda_{m}=2 \pi / k_{\text {imag }}$, and phase velocity, $v_{\text {phase }}=$ $\omega / k_{\text {imag. }}$. With no absorption $\left(\beta=0\right.$ so that $\left.k_{\text {real }}=k_{\text {imag }}\right)$, photon-density waves are attenuated to $0.2 \%(27 \mathrm{~dB})$ of their initial amplitude in just one modulation wavelength. For typical biological tissues and modulation frequencies (200 $\mathrm{MHz}, \beta=0.1 / \mathrm{cm}, \sigma_{\mathrm{tr}}=20 / \mathrm{cm}, n=1.40$ ), photondensity waves are attenuated by 10 orders of magnitude (101 dB) in one modulation wavelength.

Experimentally the source consists of a dc term plus an ac term, $S=S_{\mathrm{dc}}+S_{\mathrm{ac}} \exp (i \omega t)$, so the fluence rate has a similar form:

$$
\begin{aligned}
\phi(\mathbf{r}, t)= & A_{\mathrm{dc}} \frac{\exp (-r / \delta)}{r}+A_{\mathrm{ac}} \frac{\exp \left(-k_{\text {real }} r\right)}{r} \\
& \times \exp \left[-i\left(k_{\text {imag }} r-\omega t\right)\right],
\end{aligned}
$$

where the dc attenuation length $\delta$ is defined by $k_{\text {real }}(\omega=$ $0)=1 / \delta=\sqrt{3 \beta \sigma_{\text {tr }}}$.

A detector placed a distance $r$ away from the source will measure the radiance $L(\mathbf{r}, \hat{s}, t)$, which depends on the orientation $\hat{s}$ of the detector [see Eq. (2.1.4)]. Experimentally our detector fiber is oriented perpendicular to the radial flux from the source (Fig. 1), so the detector signal is simply proportional to the fluence rate $\phi(r, t)$ given in Eq. (2.2.5). The detector then observes the photondensity wave with a phase lag relative to the source (at $r=0$ ) given by

$$
\text { phase lag }=k_{\mathrm{imag}} r=\sqrt{\frac{3}{2} \beta \sigma_{\mathrm{tr}}}\left[\sqrt{1+(\omega \tau)^{2}}-1\right]^{1 / 2} r
$$

The modulation amplitude of the wave relative to that at the source $(r=0)$ will be

$$
\begin{aligned}
\text { modulation } & =\frac{\left[A_{\mathrm{ac}} \frac{\exp \left(-k_{\mathrm{real}} r\right)}{r} / A_{\mathrm{dc}} \frac{\exp (-r / \delta)}{r}\right]}{\left[A_{\mathrm{ac}} / A_{\mathrm{dc}}\right]} \\
& =\exp \left[-\left(k_{\text {real }}-\frac{1}{\delta}\right) r\right]=\exp \left\{-\sqrt{\frac{3}{2} \beta \sigma_{\mathrm{tr}}}\right. \\
& \left.\times\left[\left[\sqrt{1+(\omega \tau)^{2}}+1\right]^{1 / 2}-\sqrt{2}\right] r\right\} \cdot(2.2 .7)
\end{aligned}
$$




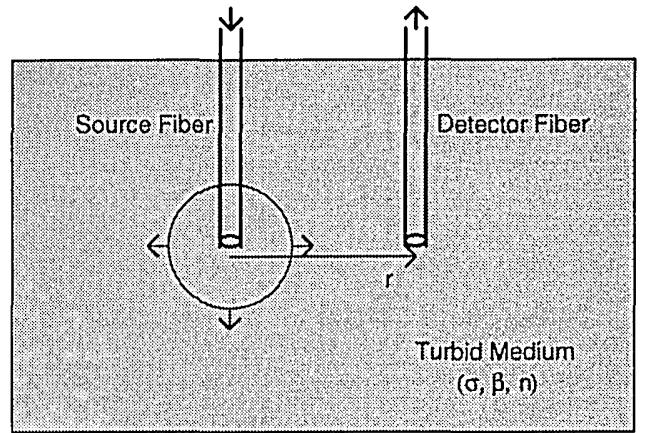

Fig. 1. Infinite-medium geometry. The medium is strongly scattering with scattering coefficient $\sigma$, absorption coefficient $\beta$, and refractive index $n$. The detector fiber is oriented perpendicular to the radial flux from the source, so the detector signal is simply proportional to the fluence rate.

Table 1. Values for the Ratio of the Fluence Rate to the Flux in an Infinite Medium ${ }^{a}$

\begin{tabular}{cclcc}
\hline $\begin{array}{c}\text { Frequency } \\
(\mathrm{MHz})\end{array}$ & $\begin{array}{c}k_{\text {real }} \\
(1 / \mathrm{cm})\end{array}$ & $\begin{array}{c}k_{\text {imag }} \\
(1 / \mathrm{cm})\end{array}$ & $\begin{array}{c}\lambda_{m} \\
(\mathrm{~cm})\end{array}$ & $|\phi| / 3|\mathrm{j}|$ \\
\hline 0 & 2.45 & 0 & $\infty$ & 8.2 \\
100 & 2.47 & 0.355 & 17.7 & 8.0 \\
200 & 2.55 & 0.692 & 9.08 & 7.6 \\
500 & 2.88 & 1.53 & 4.11 & 6.1 \\
1000 & 3.51 & 2.51 & 2.50 & 4.6 \\
\hline
\end{tabular}

${ }^{a}$ Photon-density plane waves were considered in a turbid medium with $\beta=0.1 / \mathrm{cm}, \sigma_{\mathrm{tr}}=20 / \mathrm{cm}$, and $n=1.40$. Equation (2A.6) was used to evaluate the flux.

It is important to check the validity of the diffusion approximation [Eq. (2.1.4)], which we used to derive the diffusion equation [Eq. (2.1.8)]. Specifically, we want to see whether the amplitude of the isotropic fluence rate $\phi$ is much larger than the directional asymmetry term $3|\mathbf{j}|$. In Table 1 we list the ratio $|\phi| / 3|\mathbf{j}|$ for typical biological tissue at a number of common modulation frequencies. As expected, increasing the modulation frequency results in a decrease in modulation wavelength $\lambda_{m}$ and in the ratio $|\phi| / 3|\mathbf{j}|$. Values for $|\phi| / 3|\mathbf{j}|$ range from 8.2 to 4.6 , indicating the tentative validity of the diffusion approximation in the dc-to- $1-\mathrm{GHz}$ region. Strictly speaking, the upper frequency limit for diffusion validity should be imposed by the reciprocal of the mean time $\tau_{\mathrm{sc}}$ between scattering events, i.e., $\omega \ll 1 / \tau_{\mathrm{sc}}=\sigma_{\mathrm{tr}} c \approx 430 \mathrm{GHz}$. However, in practical terms the frequency-dependent reduction in $|\phi| / 3|\mathbf{j}|$ imposes an upper limit in the tensof-gigahertz region.

\section{Partial-Current Boundary Condition for a}

\section{Semi-Infinite Medium}

In noninvasive clinical applications of FDPM the source and the detector must be placed on the surface of the tissue to be examined. Perhaps the most common FDPM geometry can be approximated by a semi-infinite medium with a planar boundary (Fig. 2). Solutions to diffusion equation (2.1.8) are then subject to a boundary condition at the planar surface $(z=0)$. If the boundary is perfectly transmitting (no Fresnel reflection), then the radiance $L(\mathbf{r}, \hat{s})$ evaluated at the boundary must fall to zero when the direction $\hat{s}$ points into the medium from outside. This constraint results in a discontinuity in the ra- diance as $\hat{s}$ varies in direction from pointing out of the medium to pointing into the medium. The discontinuity is a clear violation of the diffusion approximation expressed in Eq. (2.1.4), in which the radiance is assumed to be primarily isotropic with a small directional flux.

However, if the refractive indices of the strongly scattering medium and of the bounding transparent medium are substantially different (e.g., $n=1.40$ and $n_{\text {out }}=$ 1.00 ), a significant fraction of the radiant energy incident upon the boundary from inside will be reflected back into the turbid medium. This reflected radiance is effectively equivalent to radiant energy incident upon the medium from outside. Since radiant energy incident from inside at a grazing angle will suffer total internal reflection, the radiance $L(\mathbf{r}, \hat{s})$ can now vary smoothly as $\hat{s}$ changes direction from pointing outside to pointing inside the medium, and the discontinuity associated with the perfectly transmitting boundary condition is avoided. In our solution to diffusion equation (2.1.8) we shall see that the extent of anisotropy in $L(\mathbf{r}, \hat{s})$ at the boundary is specified in the boundary condition.

Diffusion equation (2.1.8) involves the radiant-energy fluence rate $\phi(\mathbf{r}, t)$, which is the integral of the radiance over all solid angles, so we shall adopt a boundary condition expressed in terms of the fluence rate rather than in terms of the radiance in a particular direction $\hat{s}$. Following the partial-current treatment of Keijzer et al. ${ }^{10}$ (see also Refs. 11 and 12), we set the irradiance at the boundary equal to the integral of the reflected radiance:

$$
E_{\text {irrad }}=\iint_{\hat{s} \cdot \hat{n}>0} R_{\text {Fresnel }}(\hat{s}) L(\hat{s}) \hat{s} \cdot \hat{n} \mathrm{~d} \Omega,
$$

where $\hat{n}=-\hat{z}$ is the outward-drawn normal to the boundary (see Fig. 2 ) and $R_{\text {Fresnel }}(\hat{s})$ is the Fresnel reflection coefficient for light incident upon the boundary in a direction $\hat{s}$ from within the medium. We use the Fresnel reflection coefficient for unpolarized light:

$$
\begin{aligned}
& R_{\text {Fresnel }}(\theta)=\frac{1}{2}\left(\frac{n \cos \theta^{\prime}-n_{\text {out }} \cos \theta}{n \cos \theta^{\prime}+n_{\text {out }} \cos \theta}\right)^{2} \\
& +\frac{1}{2}\left(\frac{n \cos \theta-n_{\text {out }} \cos \theta^{\prime}}{n \cos \theta+n_{\text {out }} \cos \theta^{\prime}}\right)^{2} \\
& \text { when } 0 \leq \theta \leq \theta_{c} \text {, } \\
& =1 \quad \text { when } \theta_{c} \leq \theta \leq \pi / 2 \text {, }
\end{aligned}
$$

where the angle of incidence $\theta$ from within the medium is given by $\cos \theta=\hat{s} \cdot \hat{n}$, the refracted angle $\theta^{\prime}$ in the

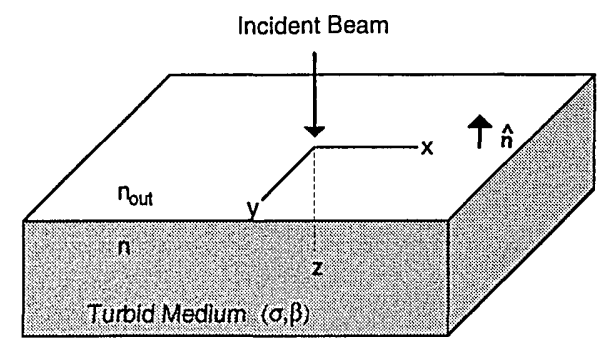

Fig. 2. Semi-infinite-medium geometry. The lower medium is strongly scattering, with scattering coefficient $\sigma$, absorption coefficient $\beta$, and refractive index $n$. The upper medium is transparent, with refractive index $n_{\text {out }} . \hat{n}$ is the outward-drawn normal to the boundary. 
outside medium satisfies $n \sin \theta=n_{\text {out }} \sin \theta^{\prime}$, and the critical angle $\theta_{c}$ for total internal reflection is given by $n \sin \theta_{c}=n_{\text {out }}$. The irradiance on the left-hand side of Eq. (2.3.1) can be evaluated with Eq. (2.1.4):

$$
E_{\text {irrad }}=\iint_{\hat{s} \cdot \hat{n}<0} L(\hat{s}) \hat{s} \cdot(-\hat{n}) \mathrm{d} \Omega=\frac{\phi}{4}+\frac{j_{z}}{2},
$$

and the integrated reflected radiance on the right-hand side of Eq. (2.3.1) can be written with Eq. (2.1.4):

$$
\iint_{\hat{s} \cdot \hat{n}>0} R_{\text {Fresnel }}(\hat{s}) L(\hat{s}) \hat{s} \cdot \hat{n} \mathrm{~d} \Omega=R_{\phi} \frac{\phi}{4}-R_{j} \frac{j_{z}}{2},
$$

where

$$
\begin{aligned}
& R_{\phi} \equiv \int_{0}^{\pi / 2} 2 \sin \theta \cos \theta R_{\text {Fresnel }}(\theta) \mathrm{d} \theta \\
& R_{j} \equiv \int_{0}^{\pi / 2} 3 \sin \theta \cos ^{2} \theta R_{\text {Fresnel }}(\theta) \mathrm{d} \theta .
\end{aligned}
$$

So boundary condition (2.3.1) reduces to

$$
\frac{\phi}{4}+\frac{j_{z}}{2}=R_{\phi} \frac{\phi}{4}-R_{j} \frac{j_{z}}{2} \quad \text { or } \phi=\frac{1+R_{j}}{1-R_{\phi}}\left(-2 j_{z}\right) \text {. }
$$

Notice that the boundary condition specifies the ratio of the fluence rate to the normal component of the flux at the surface, and we expect this ratio to be large if the diffusion approximation is to be valid. We rewrite Eq. (2.3.6) and define an effective reflection coefficient $R_{\text {eff }}$ :

$$
\frac{|\phi|}{3\left|j_{z}\right|}=\frac{2}{3} \frac{1+R_{j}}{1-R_{\phi}} \equiv \frac{2}{3} \frac{1+R_{\text {eff }}}{1-R_{\text {eff }}}
$$

or

$$
R_{\text {eff }} \equiv \frac{R_{\phi}+R_{j}}{2-R_{\phi}+R_{j}}
$$

The effective reflection coefficient $R_{\text {eff }}$ is a composite of flux $\left(R_{j}\right)$ and fluence rate $\left(R_{\phi}\right)$ terms and represents the fraction of the emittance that is reflected and becomes the irradiance:

$$
\begin{aligned}
E_{\mathrm{irrad}} & =R_{\text {eff }} E_{\text {emitt }}=R_{\text {eff }} \iint_{\hat{s} \cdot \hat{n}>0} L(\hat{s}) \hat{s} \cdot \hat{n} \mathrm{~d} \Omega \\
& =R_{\text {eff }}\left(\frac{\phi}{4}-\frac{j_{z}}{2}\right) .
\end{aligned}
$$

The ratio $|\phi| / 3\left|j_{z}\right|$ in Eq. (2.3.7) was evaluated for a perfectly transmitting boundary and for two realistic mismatches in refractive indices; the results are presented in Table 2. For typical source-detector geometries (see Figs. 2 and 3), there will be a radial component of the flux as well as a normal component, especially near the source. However, at distances greater than $10 l_{\text {tr }}$ the ratio $|\phi| / 3\left|j_{z}\right|$ will be only a slight underestimate of the anisotropy in the radiance at the boundary.

Note in Table 2 that the presence of a Fresnel reflection increases the ratio from an unacceptable value of $2 / 3$ for the perfectly transmitting boundary to a marginal ratio of 2 for an air-tissue interface. Recall from Table 1 that in an infinite medium the ratio is roughly 7 , so the presence of the boundary strains the diffusion approximation [Eq. (2.1.4)] even further.

\section{Image Configuration for the Partial-Current Boundary Condition}

The partial-current boundary condition expressed in Eqs. (2.3.1), (2.3.6), and (2.3.7) can be written in terms of the fluence rate and its normal derivative with the help of Eq. (2.1.6):

$$
\phi=l_{s} \frac{\partial \phi}{\partial z} \quad \text { at } z=0
$$

where

$$
l_{s} \equiv \frac{1+R_{\text {eff }}}{1-R_{\text {eff }}} 2 D=\frac{1+R_{\text {eff }}}{1-R_{\text {eff }}} \frac{2}{3} l_{\text {tr }} .
$$

This mixed Dirichlet-Neuman boundary condition has been applied to radiative transfer theory by several authors. ${ }^{10-12}$

We mention in passing that, at the interface between two different turbid media, the boundary condition commonly used is simply the continuity of the fluence rate $\phi$ and the normal component of the flux $D(\partial \phi / \partial n){ }^{4,16}$ These separate Dirichlet and Neuman boundary conditions hold only in the absence of Fresnel reflection. Using the method of Subsection 2.C, we have taken account of Fresnel reflection and find that, while the normal component of the flux is still continuous $\left(j_{z}^{(1)}=j_{z}^{(2)} \equiv j_{z}\right)$, the fluence rate $\phi$ has a discontinuity given by (see Fig. $2, n \rightarrow n_{1}$ and $n_{\text {out }} \rightarrow n_{2}$ ) $\left(1-R_{12}\right) \phi^{(1)}+2\left(R_{12}-R_{21}\right) j_{z}=\left(1-R_{21}\right) \phi^{(2)}$. In this expression, $R_{12}$ is the effective reflection coefficient [see Eq. (2.3.7)] for radiation incident from medium 1 upon medium 2, and vice versa for $R_{21}$.

In addition to specifying a boundary condition at $z=0$, we must choose a form for the source term $S(\mathbf{r}, t)$ in diffusion equation (2.1.8). In this semi-infinite geometry the source is typically a laser beam incident upon the medium along the $z$ axis. The points at which the first scattering events occur are distributed exponentially into the medium. The source can be modeled as a line

Table 2. Values for the Ratio of the Fluence Rate to the Flux at the Surface

for Typical Mismatches in Refractive Indices

\begin{tabular}{lllllc}
\hline$n$ & $n_{\text {out }}$ & \multicolumn{1}{c}{$R_{\phi}$} & \multicolumn{1}{c}{$R_{j}$} & \multicolumn{1}{c}{$R_{\text {eff }}$} & $|\phi| / 3\left|j_{z}\right|$ \\
\hline 1.00 & 1.00 & 0 & 0 & 0 & 0.7 \\
1.33 & 1.00 & 0.472 & 0.328 & 0.431 & 1.7 \\
1.40 & 1.00 & 0.529 & 0.389 & 0.493 & 2.0 \\
\hline
\end{tabular}

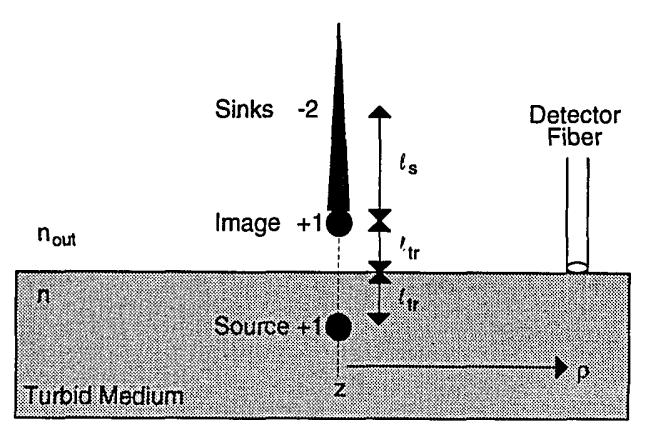

Fig. 3. Source, image, and continuous line of sinks that constitute the fluence rate solution in a semi-infinite medium with the partial-current boundary condition expressed in Eq. (2.4.1). 
of isotropic point sources with strengths that are exponentially damped according to $\exp \left(-\sigma_{\mathrm{tr}} z\right)$. We make the further approximation that the line of sources can be replaced with a single isotropic point source located a transport mean free path $l_{\mathrm{tr}}$ into the medium.

The Green's function solution to diffusion equation (2.1.8) with the partial-current boundary condition [Eq. (2.1.1)] was given by Bryan ${ }^{23}$ for a source pulse of unit energy emitted at $t=t^{\prime}$ from a point $\left(\rho=0, z=l_{\text {tr }}\right)$ (see also Subsecs. 14.2 and 14.9 of Ref. 22):

$$
\begin{aligned}
& \phi_{G}\left(\rho, z, t-t^{\prime}\right) \\
& =\frac{c}{\left[4 \pi D c\left(t-t^{\prime}\right)\right]^{3 / 2}} \exp \left[-\frac{\rho^{2}}{4 D c\left(t-t^{\prime}\right)}-\beta c\left(t-t^{\prime}\right)\right] \\
& \quad \times\left\{\exp \left[-\frac{\left(z-l_{\mathrm{tr}}\right)^{2}}{4 D c\left(t-t^{\prime}\right)}\right]+\exp \left[-\frac{\left(z+l_{\mathrm{tr}}\right)^{2}}{4 D c\left(t-t^{\prime}\right)}\right]\right. \\
& \left.-\frac{2}{l_{s}} \int_{0}^{\infty} \mathrm{d} l \exp \left(-l / l_{s}\right) \exp \left[-\frac{\left(z+l_{\mathrm{tr}}+l\right)^{2}}{4 D c\left(t-t^{\prime}\right)}\right]\right\} \cdot(2.4 .2)
\end{aligned}
$$

Bryan $^{23}$ used the method of images to construct this Green's function, and he emphasized the following interpretation of the three terms in the sum that compose the solution: the first term is the response to the original point source at $\left(\rho=0, z=l_{\text {tr }}\right.$ ), the second is due to an image source of the same sign and magnitude at ( $\rho=0$, $z=-l_{\text {tr }}$ ), and the third represents a continuous line of sinks stretching from $\left(\rho=0, z=-l_{\text {tr }}\right)$ to $(\rho=0, z=-\infty)$ (see Fig. 3). The sinks are damped in amplitude according to $\exp \left(-l / l_{s}\right)$, where $l$ is measured from the image source at $\left(\rho=0, z=-l_{\text {tr }}\right)$. The total strength of the sinks is twice that of either the original source or the image source, but the sign of the sinks is negative.

One of the major points of this paper is that Bryan's solution provides a convenient interpretation of the fluence rate for a semi-infinite medium in terms of image sources and sinks. This interpretation remains valid when the isotropic delta function source at $(\rho=0, z=$ $l_{\text {tr }}$ ) is replaced with a harmonic source emitting power $P \exp (i \omega t)$. The fluence rate solution becomes

$$
\begin{aligned}
& \phi_{\omega}(\rho, z, t)=\int_{-\infty}^{t} \phi_{G}\left(\rho, z, t-t^{\prime}\right) P \exp \left(i \omega t^{\prime}\right) \mathrm{d} t^{\prime} \\
&=\frac{P \exp (i \omega t)}{4 \pi D}\left(\frac{\exp \left(-k r_{1}\right)}{r_{1}}+\frac{\exp \left(-k r_{2}\right)}{r_{2}}-\frac{2}{l_{s}}\right. \\
&\left.\times \int_{0}^{\infty} \mathrm{d} l \exp \left(-l / l_{s}\right) \frac{\exp \left\{-k\left[\left(z+l_{\mathrm{tr}}+l\right)^{2}+\rho^{2}\right]^{1 / 2}\right\}}{\left[\left(z+l_{\mathrm{tr}}+l\right)^{2}+\rho^{2}\right]^{1 / 2}}\right),
\end{aligned}
$$

where

$$
r_{1}=\left[\left(z-l_{\mathrm{tr}}\right)^{2}+\rho^{2}\right]^{1 / 2}, \quad r_{2}=\left[\left(z+l_{\mathrm{tr}}\right)^{2}+\rho^{2}\right]^{1 / 2},
$$

and the complex wave number $k$ is given by Eqs. (2.2.3). Experimentally the source consists of a dc term plus an ac term, $S=S_{\mathrm{dc}}+S_{\mathrm{ac}} \exp (i \omega t)$, so the fluence rate has a similar form:

$$
\begin{aligned}
& \phi(\rho, z, t)=A_{\mathrm{dc}}\left(\frac{\exp \left(-r_{1} / \delta\right)}{r_{1}}+\frac{\exp \left(-r_{2} / \delta\right)}{r_{2}}-\frac{2}{l_{s}}\right. \\
& \left.\quad \times \int_{0}^{\infty} \mathrm{d} l \exp \left(-l / l_{s}\right) \frac{\exp \left\{-\left[\left(z+l_{\mathrm{tr}}+l\right)^{2}+\rho^{2}\right]^{1 / 2} / \delta\right\}}{\left[\left(z+l_{\mathrm{tr}}+l\right)^{2}+\rho^{2}\right]^{1 / 2}}\right) \\
& \quad+A_{\mathrm{ac}} \exp (i \omega t)\left(\frac{\exp \left(-k r_{1}\right)}{r_{1}}+\frac{\exp \left(-k r_{2}\right)}{r_{2}}-\frac{2}{l_{s}}\right. \\
& \left.\quad \times \int_{0}^{\infty} \mathrm{d} l \exp \left(-l / l_{s}\right) \frac{\exp \left\{-k\left[\left(z+l_{\mathrm{tr}}+l\right)^{2}+\rho^{2}\right]^{1 / 2}\right\}}{\left[\left(z+l_{\mathrm{tr}}+l\right)^{2}+\rho^{2}\right]^{1 / 2}}\right) .
\end{aligned}
$$

It is instructive to check the limiting behavior of the fluence rate in Eq. (2.4.5) as the boundary becomes highly reflecting, i.e., as $R_{\text {eff }} \rightarrow 1$. According to the partialcurrent boundary condition [Eq. (2.4.1)], the attenuation length $l_{s}$ of the sink amplitudes becomes infinite. However, the total strength of the line of sinks remains fixed, so the amplitude of any finite length of sinks becomes infinitesimal, and the integrals over the sinks in Eq. (2.4.5) tend to zero. In effect, the sinks become so distant that the photon-density waves that they emit $\left(180^{\circ}\right.$ out of phase with the source and its image) are too strongly damped to be significant. The remaining terms that are due to the source and its image become identical at points on the boundary $(z=0)$, and the fluence rate at the surface can be seen to be simply twice the fluence rate that is due to a single source at $\left(\rho=0, z=l_{\text {tr }}\right)$ in an infinite medium [cf. Eq. (2.2.5)]. In general, the more highly reflecting the boundary, the more the fluence rate tends toward the infinite-medium solution and the less likely it is that the diffusion approximation will be violated at the boundary.

An optical fiber placed at the surface of the semi-infinite medium intercepts the transmitted radiance integrated over the numerical aperture of the fiber (see Fig. 3). The diffusion approximation for the radiance [Eq. (2.1.4)], Fick's law [Eq. (2.1.6)], and the partial-current boundary condition [Eq. (2.4.1)] can be combined to show that the detected signal is simply proportional to the fluence rate $\phi(\rho, z=0, t)$ at the surface:

$$
\begin{aligned}
\text { signal }= & \iint_{A_{\text {fiber }}} \mathrm{d} x \mathrm{~d} y \iint_{\Omega_{\text {fiber }}} \mathrm{d} \Omega T_{\text {Fresnel }}(\hat{s}) \\
& \times L(x, y, z=0, \hat{s})(\hat{s} \cdot \hat{n}) \\
= & \iint_{A_{\text {fiber }}} \mathrm{d} x \mathrm{~d} y \iint_{\Omega_{\text {fiber }}} \mathrm{d} \Omega T_{\text {Fresnel }}(\hat{s}) \frac{1}{4 \pi} \\
& \times\left[\phi(x, y, z=0)+3 D \frac{\partial \phi}{\partial z}\right. \\
& \times(x, y, z=0) \cos \theta] \cos \theta \\
= & \iint_{A_{\text {fiber }}} \mathrm{d} x \mathrm{~d} y \iint_{\Omega_{\text {fiber }}} \mathrm{d} \Omega T_{\text {Fresnel }}(\hat{s}) \frac{1}{4 \pi} \\
& \times\left[\phi(x, y, z=0)+\frac{3}{2} \frac{\left(1-R_{\text {eff }}\right)}{\left(1+R_{\text {eff }}\right)}\right. \\
& \times \phi(x, y, z=0) \cos \theta] \cos \theta \\
\propto & \phi(x, y, z=0),
\end{aligned}
$$

where $T_{\text {Fresnel }}(\hat{s})=1-R_{\text {Fresnel }}(\hat{s})$, and $R_{\text {Fresnel }}(\hat{s})$ is given 
by Eq. (2.3.2). The phase lag and the modulation of the photon-density waves at the detector fiber can be written as [cf. Eqs. (2.2.6) and (2.2.7)]

$$
\begin{aligned}
\text { phase lag } & =k_{\text {imag }} r_{0}-\arctan (\mathrm{IMAG} / \mathrm{REAL}), \\
\text { modulation } & =\left(\mathrm{REAL}^{2}+\mathrm{IMAG}^{2}\right)^{1 / 2} / \mathrm{dc},
\end{aligned}
$$

where

$$
\begin{aligned}
\mathrm{REAL}= & \frac{\exp \left(-k_{\mathrm{real}} r_{0}\right)}{r_{0}}-\frac{1}{l_{s}} \int_{0}^{\infty} \mathrm{d} l \exp \left(-l / l_{s}\right) \\
& \times \frac{\exp \left(-k_{\mathrm{real}} r_{0 l}\right)}{r_{0 l}} \cos \left[k_{\mathrm{imag}}\left(r_{0 l}-r_{0}\right)\right], \\
\mathrm{IMAG}= & \frac{1}{l_{s}} \int_{0}^{\infty} \mathrm{d} l \exp \left(-l / l_{s}\right) \\
& \times \frac{\exp \left(-k_{\mathrm{real}} r_{0 l}\right)}{r_{0 l}} \sin \left[k_{\mathrm{imag}}\left(r_{0 l}-r_{0}\right)\right], \\
\mathrm{dc}= & \frac{\exp \left(-r_{0} / \delta\right)}{r_{0}}-\frac{1}{l_{s}} \int_{0}^{\infty} \mathrm{d} l \exp \left(-l / l_{s}\right) \frac{\exp \left(-r_{0 l} / \delta\right)}{r_{0 l}}, \\
r_{0}= & \left(l_{\mathrm{tr}}^{2}+\rho^{2}\right)^{1 / 2}, \quad r_{0 l}=\left[\left(l_{\mathrm{tr}}+l\right)^{2}+\rho^{2}\right]^{1 / 2} .(2.4 .8)
\end{aligned}
$$

When the boundary is perfectly reflecting, Eqs. (2.4.7) reduce to the infinite-medium expressions for the phase and the modulation [Eqs. (2.2.6) and (2.2.7), respectively].

\section{E. Zero-Boundary Condition}

We have referred to Eq. (2.4.1) as the partial-current boundary condition, ${ }^{10}$ although it is also known as the radiation boundary condition in the context of heat diffusion. ${ }^{22}$ Two other boundary conditions have been used in solving diffusion equation (2.1.8) in radiative transfer. The first sets the fluence rate equal to zero at the physical boundary, $\phi(\rho, z=0)=0 . .^{21,24}$ Although this condition is unphysical and violates the diffusion approximation, it is mathematically simple, and some researchers have argued that it is a sufficiently good approximation for biological tissues. ${ }^{25}$ This zero boundary condition can be satisfied by introduction of a negative image at $z=-l_{\mathrm{tr}}$, yielding the following expression for the fluence rate:

$$
\begin{array}{r}
\phi(\rho, z, t)=A_{\mathrm{dc}}\left[\frac{\exp \left(-r_{1} / \delta\right)}{r_{1}}-\frac{\exp \left(-r_{2} / \delta\right)}{r_{2}}\right] \\
+A_{\mathrm{ac}} \exp (i \omega t)\left[\frac{\exp \left(-k r_{1}\right)}{r_{1}}-\frac{\exp \left(-k r_{2}\right)}{r_{2}}\right],
\end{array}
$$

where

$$
r_{1}=\left[\left(z-l_{\mathrm{tr}}\right)^{2}+\rho^{2}\right]^{1 / 2}, \quad r_{2}=\left[\left(z+l_{\mathrm{tr}}\right)^{2}+\rho^{2}\right]^{1 / 2} .
$$

Because the fluence rate is zero at the boundary, the transmitted radiance detected at the surface has a contribution only from the flux [see Eq. (2.1.4)]:

$$
\begin{aligned}
\text { signal }= & \iint_{A_{\text {fiber }}} \mathrm{d} x \mathrm{~d} y \iint_{\Omega_{\text {fiber }}} \mathrm{d} \Omega T_{\text {Fresnel }}(\hat{s}) \\
& \times L(x, y, z=0, \hat{s})(\hat{s} \cdot \hat{n}) \\
= & \iint_{A_{\text {fiber }}} \mathrm{d} x \mathrm{~d} y \iint_{\Omega_{\text {fiber }}} \mathrm{d} \Omega T_{\text {Fresnel }}(\hat{s}) \\
& \times \frac{3}{4 \pi} D \frac{\partial \phi}{\partial z}(x, y, z=0) \cos ^{2} \theta \\
\propto & \frac{\partial \phi}{\partial z}(x, y, z=0) .
\end{aligned}
$$

The phase lag and the modulation of the detected signal can be expressed as

$$
\begin{aligned}
\text { phase lag }= & k_{\text {imag }} r_{0}-\arctan \left(\frac{k_{\text {imag }}}{k_{\text {real }}+1 / r_{0}}\right), \\
\text { modulation }= & \frac{\left[\left(k_{\text {real }}+1 / r_{0}\right)^{2}+k_{\text {imag }}^{2}\right]^{1 / 2}}{\left(1 / \delta+1 / r_{0}\right)} \\
& \times \exp \left[-\left(k_{\text {real }}-1 / \delta\right) r_{0}\right] .
\end{aligned}
$$

Note that Eqs. (2.5.4) do not account for a refractive-index mismatch at the boundary.

\section{F. Extrapolated-Boundary Condition}

Moulton, ${ }^{6}$ Patterson et al., ${ }^{7}$ and Farrel et al. ${ }^{8}$ have employed a more palatable boundary condition in which the fluence rate is set equal to zero at an extrapolated boundary located a distance $z_{b}$ outside the turbid medium, $\phi\left(\rho, z=-z_{b}\right)=0$. This extrapolatedboundary approach has its origin in the rigorous solution of the Milne problem (see Subsecs. 5.6 and 6.4 of Ref. 13; Chap. 4, Subsec. IV.E of Ref. 14; and Subsecs. 5.39-5.42 of Ref. 16). The Milne problem involves the solution of the time-independent transport equation for a semi-infinite medium with a source located infinitely deep inside the medium. The fluence rate solution to the Milne problem is nonzero on the boundary but extrapolates to zero at a distance $z_{b}$ outside the medium. Moulton ${ }^{6}$ modified Marshak's boundary condition for the $P_{1}$ approximation (see Subsec. 10.5 of Ref. 15) to include a Fresnel reflection at the surface, and he obtained an approximate value for $z_{b}$ :

$$
z_{b}=l_{s}=\frac{1+R_{\mathrm{eff}}}{1-R_{\mathrm{eff}}} \frac{2}{3} l_{\mathrm{tr}} .
$$

Aronson ${ }^{9}$ used numerical methods to obtain the solution to the transport equation that includes the Fresnel reflection at the boundary, and his values for $z_{b}$ are $\sim 5 \%$ smaller than those of Eq. (2.6.1) when the refractive-index mismatch is air-water or air-flesh.

The method of images can be used to construct a fluence rate solution that satisfies $\phi\left(\rho, z=-z_{b}\right)=0$. The resulting configuration of images is depicted in Fig. 4 , and

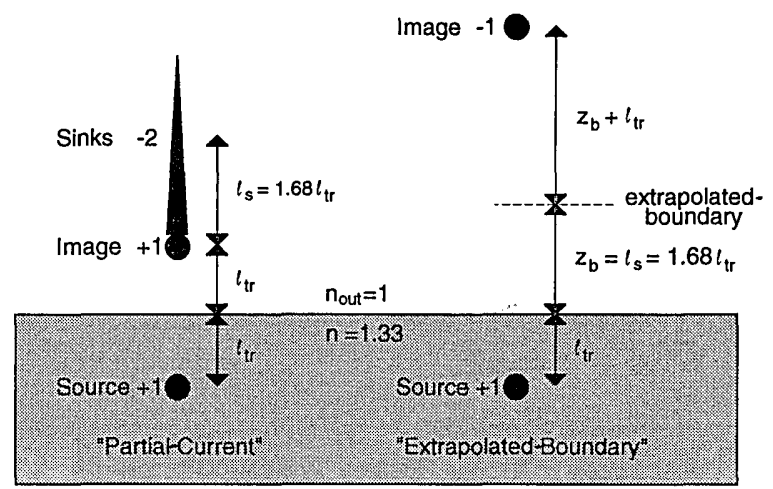

Fig. 4. Source and image configurations for two different boundary conditions: partial-current at the left and extrapolated boundary at the right. The placement of the images is scaled appropriately for an air-medium interface, with the refractive index of the medium equal to that of water. 
the associated expression for the fluence rate is

$$
\begin{array}{r}
\phi(\rho, z, t)=A_{\mathrm{dc}}\left[\frac{\exp \left(-r_{1} / \delta\right)}{r_{1}}-\frac{\exp \left(-r_{b} / \delta\right)}{r_{b}}\right] \\
+A_{\mathrm{ac}} \exp (i \omega t)\left[\frac{\exp \left(-k r_{1}\right)}{r_{1}}-\frac{\exp \left(-k r_{b}\right)}{r_{b}}\right],
\end{array}
$$

oriented normal to the boundary and that the numerical aperture of the fiber is small, then the following expressions describe the phase lag and modulation of the photon density waves at the detector:

$$
\begin{aligned}
\text { phase lag } & =k_{\text {imag }} r_{0}-\arctan (\mathrm{IMAG} / \mathrm{REAL}), \\
\text { modulation } & =\left(\mathrm{REAL}^{2}+\mathrm{IMAG}^{2}\right)^{1 / 2} / \mathrm{dc},
\end{aligned}
$$

where

$$
\begin{aligned}
& \mathrm{REAL}=\frac{\exp \left(-k_{\text {real }} r_{0}\right)}{r_{0}}-\cos \left[k_{\text {imag }}\left(r_{0 b}-r_{0}\right)\right] \frac{\exp \left(-k_{\text {real }} r_{0 b}\right)}{r_{0 b}}+\left(k_{\text {real }}+\frac{1}{r_{0}}\right) \frac{l_{\text {tr }}^{2}}{r_{0}} \frac{\exp \left(-k_{\text {real }} r_{0}\right)}{r_{0}}+\left(k_{\text {real }}+\frac{1}{r_{0 b}}\right) \\
& \times \frac{\left(2 z_{b}+l_{\mathrm{tr}}\right) l_{\mathrm{tr}}}{r_{0 b}} \cos \left[k_{\text {imag }}\left(r_{0 b}-r_{0}\right)\right] \frac{\exp \left(-k_{\mathrm{real}} r_{0 b}\right)}{r_{0 b}}+k_{\text {imag }} \frac{\left(2 z_{b}+l_{\mathrm{tr}}\right) l_{\mathrm{tr}}}{r_{0 b}} \sin \left[k_{\text {imag }}\left(r_{0 b}-r_{0}\right)\right] \frac{\exp \left(-k_{\text {real }} r_{0 b}\right)}{r_{0 b}}, \\
& \mathrm{IMAG}=\sin \left[k_{\text {imag }}\left(r_{0 b}-r_{0}\right)\right] \frac{\exp \left(-k_{\text {real }} r_{0 b}\right)}{r_{0 b}}+k_{\text {imag }} \frac{l_{\text {tr }}^{2}}{r_{0}} \frac{\exp \left(-k_{\text {real }} r_{0}\right)}{r_{0}}+k_{\text {imag }} \frac{\left(2 z_{b}+l_{\text {tr }}\right) l_{\text {tr }}}{r_{0 b}} \cos \left[k_{\text {imag }}\left(r_{0 b}-r_{0}\right)\right] \\
& \times \frac{\exp \left(-k_{\text {real }} r_{0 b}\right)}{r_{0 b}}-\left(k_{\text {real }}+\frac{1}{r_{0 b}}\right) \frac{\left(2 z_{b}+l_{\text {tr }}\right) l_{\text {tr }}}{r_{0 b}} \sin \left[k_{\text {imag }}\left(r_{0 b}-r_{0}\right)\right] \frac{\exp \left(-k_{\text {real }} r_{0 b}\right)}{r_{0 b}}, \\
& \mathrm{dc}=\frac{\exp \left(-r_{0} / \delta\right)}{r_{0}}-\frac{\exp \left(-r_{0 b} / \delta\right)}{r_{0 b}}+\left(\frac{1}{\delta}+\frac{1}{r_{0}}\right) \frac{l_{\mathrm{tr}}^{2}}{r_{0}} \frac{\exp \left(-r_{0} / \delta\right)}{r_{0}}+\left(\frac{1}{\delta}+\frac{1}{r_{0 b}}\right) \frac{\left(2 z_{b}+l_{\mathrm{tr}}\right) l_{\mathrm{tr}}}{r_{0 b}} \frac{\exp \left(-r_{0 b} / \delta\right)}{r_{0 b}}, \\
& \left.r_{0}=\left(l_{\mathrm{tr}}^{2}+\rho^{2}\right)^{1 / 2}, \quad r_{0 b}=\left(2 z_{b}+l_{\mathrm{tr}}\right)^{2}+\rho^{2}\right]^{1 / 2} \text {. }
\end{aligned}
$$

$r_{1}=\left[\left(z-l_{\mathrm{tr}}\right)^{2}+\rho^{2}\right]^{1 / 2}, \quad r_{b}=\left[\left(z+2 z_{b}+l_{\mathrm{tr}}\right)^{2}+\rho^{2}\right]^{1 / 2}$.

The extrapolated boundary and the partial-current configurations are depicted side by side in Fig. 4, and it is interesting to note that the two configurations have the same dipole and quadrupole moments. The dipole moments are $p=2\left(l_{s}+l_{\text {tr }}\right)$, and the quadrupole moments are $Q=8 l_{s}\left(l_{s}+l_{\text {tr }}\right)$ when they are evaluated with respect to an origin on the boundary. The two configurations differ only in octupole and higher moments.

In the extrapolated boundary condition the fluence rate is nonzero at the physical boundary $(z=0)$, and so the detected signal has contributions from both the fluence rate and the flux [see Eq. (2A.4)]:

$$
\begin{aligned}
\text { signal }= & \iint_{A_{\text {fiber }}} \mathrm{d} x \mathrm{~d} y \iint_{\Omega_{\text {fiber }}} \mathrm{d} \Omega T_{\text {Fresnel }}(\hat{s}) \\
& \times L(x, y, z=0, \hat{s})(\hat{s} \cdot \hat{n}) \\
= & \iint_{A_{\text {fiber }}} \mathrm{d} x \mathrm{~d} y \iint_{\Omega_{\text {fiber }}} \mathrm{d} \Omega T_{\text {Fresnel }}(\hat{s}) \\
& \times \frac{1}{4 \pi}[\phi(x, y, z=0) \\
& \left.+3 D \frac{\partial \phi}{\partial z}(x, y, z=0) \cos \theta\right] \cos \theta .
\end{aligned}
$$

The fluence rate term seems to have been neglected by some researchers, ${ }^{6-8}$ though according to the diffusion approximation it is substantially larger than the flux term. In integration of the radiance over the solid angle accepted by the fiber, the angular dependence of the flux term is, in principle, different from that of the fluence rate. This difference in angular dependence leads to an awkward result in the extrapolated-boundary approach: the precise linear combination of fluence rate and flux contained in the detected signal is a function of the numerical aperture of the fiber. If we assume that the detector fiber is
We mention in passing that Allen and McKenzie ${ }^{26}$ collapsed the extrapolated boundary image configuration of Fig. 4 above to a point dipole located on the extrapolated boundary. At short distances from the source, however, the separation of the charges in Fig. 4 should be discernible, and their expression for the fluence rate is not substantially simpler than Eq. (2.6.2).

\section{G. Comparison of the Three Boundary Conditions}

It is interesting to compare the predictions of the three boundary conditions: the partial-current [relations (2.4.7) and (2.4.8)], the extrapolated-boundary [Eqs. (2.6.5)], and the zero-boundary [(Eqs. (2.5.4)]. We have simulated phase and modulation data for these three boundary conditions, using optical properties typical of biological tissue. The data are plotted in Fig. 5 along with phase and modulation data for an infinite medium. The most striking feature of Fig. 5 is the difference between the infinite-medium curve and the three semi-infinite curves. It is obviously important to apply some boundary condition when analyzing phase and modulation data taken at the surface of tissue. On the other hand, the partial-current and extrapolated-boundary curves are almost indistinguishable, and the zero boundary curve lies just noticeably lower in phase and higher in modulation.

It is clear from the different curves of simulated data in Fig. 5 that different values for the scattering and absorption coefficients would be deduced from experimental data, depending on which boundary condition is employed in the fitting function. We have highlighted these differences by fitting the three sets of semi-infinite data in Fig. 5 with a partial-current fitting function. We also fitted the partial-current simulated data with an infinite-medium fitting function to evaluate the error introduced by failing to employ any boundary condition at all. The results are presented in Table 3. Without any boundary condition, $\sigma_{\mathrm{tr}}$ is underestimated by $20 \%$ and $\beta$ is overestimated by $50 \%$. The loss of photons through the boundary is mistaken for increased absorption in the 


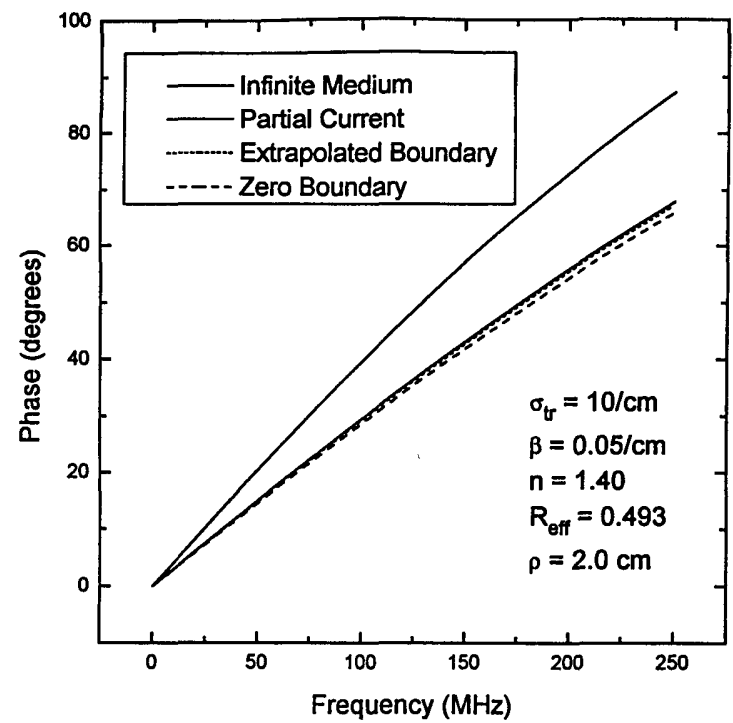

A

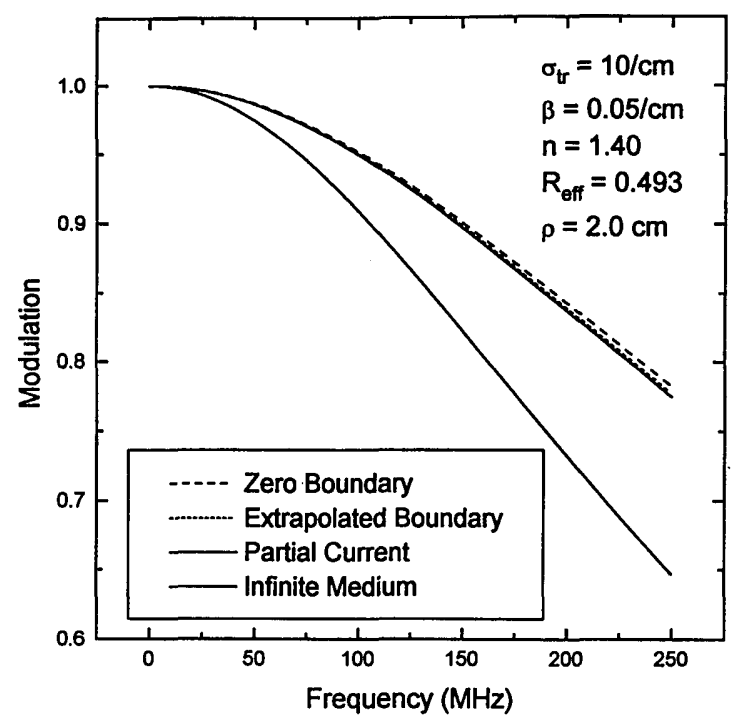

B

Fig. 5. Simulated phase (A) and modulation (B) data for an infinite medium (solid curves) and for a semi-infinite medium with three boundary conditions: partial-current, extrapolated boundary, and zero boundary. The transport scattering coefficient is $10 / \mathrm{cm}$, the absorption coefficient is $0.05 / \mathrm{cm}$, the refractive index of the turbid medium is 1.40 , the effective reflection coefficient is 0.493 , and the source-detector separation is $2.0 \mathrm{~cm}$.

turbid medium. However, the three different boundary conditions yield values for $\sigma_{\operatorname{tr}}$ and $\beta$ that are the same to within $5 \%$.
In a similar way we have investigated differences in simulated data as parameter values are varied over the following ranges: $10 / \mathrm{cm} \leq \sigma_{\mathrm{tr}} \leq 50 / \mathrm{cm}, 0.03 / \mathrm{cm} \leq \beta \leq$ $0.15 / \mathrm{cm}, 1.33 \leq n \leq 1.40$ and $0.431 \leq R_{\text {eff }} \leq 0.493$, and $1 \mathrm{~cm} \leq \rho \leq 5 \mathrm{~cm}$. We found that deduced values of optical parameters differ by less than $3 \%$ when data generated with the partial-current and the extrapolatedboundary conditions are fitted, whereas the zeroboundary condition yields discrepancies up to $14 \%$. In all cases the greatest deviations occur when the sourcedetector separation $\rho$ is reduced to $1 \mathrm{~cm}$; variations in $\sigma_{\text {tr }}, \beta, n$, and $R_{\text {eff }}$ have only minor effects.

We also report that neglecting the fluence rate contribution to the detected signal in the extrapolated-boundary approach makes essentially no difference in the simulated data. Although they are not specifically contained in the boundary condition, the fluence rate and the flux at the physical boundary are evidently very nearly proportional (as required by the partial-current boundary condition), so that the signal is proportional to either the fluence rate or the flux. In Subsection 2.H we elaborate on this close similarity between solutions satisfying the partial-current and the extrapolated-boundary conditions.

H. Partial-Current-Extrapolated Boundary Unification It is clear from the preceding sections that the zero boundary condition is less attractive than the partialcurrent and extrapolated boundary conditions. The zero boundary condition maximally violates the diffusion approximation and does not account for a mismatch in refractive index. Furthermore, its use in fitting data may result in errors in optical parameters of $10-15 \%$ (when $\rho / l_{\mathrm{tr}} \approx 10$ ), a discouraging prospect at the outset of a diagnostic procedure. Accordingly, we exclude the zero boundary condition from serious consideration and focus our attention on the partial-current and extrapolated boundary conditions.

Both the partial-current and the extrapolated boundary approaches are based on sound physical principles, and their theoretical development seems unflawed. The partial-current boundary condition is logically consistent within the limitations of the diffusion approximation, and the extrapolated boundary condition is motivated by a rigorous solution to the transport equation in a fairly general situation. On the other hand, each has drawbacks. The partial-current image configuration includes an infinite line of sinks, which makes nonlinear least-squares fitting of data very time consuming. However, because the partial-current boundary condition explicitly requires the flux to be proportional to the fluence rate at the surface, the detected signal at the surface is proportional to either the fluence rate or the flux. The extrapolated boundary

Table 3. Results of Nonlinear Least-Squares Fits to Simulated Semi-Infinite-Medium Data ${ }^{a}$

\begin{tabular}{llcr}
\hline Use This & To Fit Data Simulated & & \\
Fitting Function & With This Boundary Condition & $\beta(1 / \mathrm{cm})$ & $\sigma_{\mathrm{tr}}(1 / \mathrm{cm})$ \\
\hline Partial-Current & Partial-Current & 0.0500 & 10.00 \\
Partial-Current & Extrapolated Boundary & 0.0503 & 9.90 \\
Partial-Current & Zero Boundary & 0.0505 & 9.57 \\
Infinite-Medium & Partial-Current & 0.0733 & 7.78 \\
\hline
\end{tabular}

${ }^{a}$ The fitting function used a partial-current boundary condition or no boundary condition. The simulated data were the same as in Fig. 5 and were generated by use of transport scattering coefficient of $10 / \mathrm{cm}$ and an absorption coefficient of $0.05 / \mathrm{cm}$. 
condition, on the other hand, leads to an expression for the detected signal that in principle is a function of the numerical aperture of the detector fiber, certainly a logical nuisance. Clearly it would be nice to combine the advantages and avoid the disadvantages of both conditions.

An obvious course of action is to approximate the partial-current line of sinks with a few point sinks in such a way that the dipole and quadrupole moments of the image configuration are preserved. A single point sink with charge -2 placed a distance $l_{s}$ from the $+1 \mathrm{im}$ age (see Figs. 3 and 4 ) preserves the dipole moment but reduces the quadrupole moment. With two point sinks (total charge -2) there are actually an infinite number of ways to replace the line of sinks and leave the dipole and quadrupole moments intact. Perhaps the most appealing way is to divide the total sink charge of -2 into two point sinks each with charge -1 and use their positions (2 degrees of freedom) to preserve the dipole and quadrupole moments. It turns out that one point sink must be placed on top of the +1 image, thus canceling the +1 image, and the second point sink must be placed a distance $2 l_{s}+l_{\text {tr }}$ from the physical boundary (see Fig. 4). The result is precisely the extrapolatedboundary configuration.

It is now clear why the extrapolated-boundary solution seems to satisfy the partial-current boundary condition, as mentioned in Subsection 2.G. The solutions are nearly the same because the extrapolated-boundary image configuration is the best single-point image representation of the partial-current configuration, differing only in octupole and higher multipole moments. This insight suggests a considerable simplification of extrapolated boundary equations (2.6.5) and (2.6.6). Since the extrapolated boundary fluence rate in Eq. (2.6.2) also obeys the partial-current boundary condition to a good approximation, the detected signal will be proportional to either the fluence rate or the flux contribution to the transmitted radiance. Hence we can use just the fluence rate terms in Eqs. (2.6.5) and (2.6.6) and drop the flux terms. Equations (2.6.5) and (2.6.6) can thus be shortened to

$$
\begin{aligned}
\text { phase lag } & =k_{\text {imag }} r_{0}-\arctan (\mathrm{IMAG} / \mathrm{REAL}), \\
\text { modulation } & =\left(\mathrm{REAL}^{2}+\mathrm{IMAG}^{2}\right)^{1 / 2} / \mathrm{dc},
\end{aligned}
$$

where

$$
\begin{aligned}
\mathrm{REAL}= & \frac{\exp \left(-k_{\mathrm{real}} r_{0}\right)}{r_{0}}-\cos \left[k_{\mathrm{imag}}\left(r_{0 b}-r_{0}\right)\right] \\
& \times \frac{\exp \left(-k_{\mathrm{real}} r_{0 b}\right)}{r_{0 b}}, \\
\mathrm{IMAG}= & \sin \left[k_{\mathrm{imag}}\left(r_{0 b}-r_{0}\right)\right] \frac{\exp \left(-k_{\mathrm{real}} r_{0 b}\right)}{r_{0 b}}, \\
\mathrm{dc}= & \frac{\exp \left(-r_{0} / \delta\right)}{r_{0}}-\frac{\exp \left(-r_{0 b} / \delta\right)}{r_{0 b}}, \\
r_{0}= & \left(l_{\mathrm{tr}}{ }^{2}+\rho^{2}\right)^{1 / 2}, \quad r_{0 b}=\left[\left(2 z_{b}+l_{\mathrm{tr}}\right)^{2}+\rho^{2}\right]^{1 / 2}, \\
z_{b}= & l_{s}=\frac{1+R_{\mathrm{eff}}}{1-R_{\mathrm{eff}}} \frac{2}{3} l_{\mathrm{tr}} .
\end{aligned}
$$

We incorporated Eqs. (2.7.1) and (2.7.2) into a nonlinear least-squares fitting routine and found that the speed of fitting is vastly improved over that with partial-current equations (2.4.7) and (2.4.8). Moreover, the values of optical parameters deduced from fits of data differ by less than $3 \%$ from values found with the partial-current or the extrapolated boundary approach over the wide range of values used in Subsection 2.G. We recommend simplified equations (2.7.1) and (2.7.2) as a fast, accurate means of accounting for the presence of a boundary in the analysis of FDPM data.

\section{FREQUENCY-DOMAIN PHOTON-MIGRATION MEASUREMENTS}

To test the predictions of diffusion theory, we collected FDPM data from tissue phantoms with a range of scattering and absorption coefficients. The experimental setup was described in detail previously. ${ }^{27}$ Briefly, the $650-\mathrm{nm}$ beam from an argon-pumped-dye laser was modulated by a Pockels cell at $5 \mathrm{MHz}$ with harmonic content up to $250 \mathrm{MHz}$. The beam was directed onto the surface of the tissue phantom by an optical fiber, and light emitted from a point $5-25 \mathrm{~mm}$ away was collected by a detector fiber and was led to a photomultiplier tube (Hamamatsu R928). The fibers were also pushed deep into the phantom to simulate an infinite-medium geometry. A multiharmonic Fourier transform fluorometer (SLM, Urbana, Ill., Model 48000-MHF) recorded the phase and modulation of the detected light. The reference phase and modulation data were collected with the fibers joined end to end in air while the sample photomultiplier tube was attenuated with a neutral-density filter.

The tissue phantoms were 1-L mixtures of whole milk and water in a $1 \%$ agar gel. We varied the amount of milk to attain the desired transport scattering coefficient. For example, $40 \%$ milk yielded $\sigma_{\mathrm{tr}}=6 / \mathrm{cm}$. The absorption of the gels $(\beta \approx 0.02 / \mathrm{cm})$ is due primarily to the $1 \%$ agar.

Phase and modulation data from a representative gel (40\% milk) are presented in Fig. 6. Data are shown for two different values, 1.5 and $2.5 \mathrm{~cm}$, of the sourcedetector separation and for two different placements of the source and detector fibers: (1) on the surface of the gel and (2) pushed to the center of the gel to simulate an infinite-medium environment. Notice the substantial increase in phase and decrease in modulation as the fibers are moved from the surface to the center of the gel. This change was anticipated theoretically in Subsection 2.G.

The solid curves in Fig. 6 represent nonlinear leastsquares fits to the data with Eqs. (2.2.6) and (2.2.7) for the infinite-medium data and partial-current equations (2.4.7) and (2.4.8) for the surface data. Using simplified equations (2.7.1) and (2.7.2) to fit the surface data makes essentially no difference in the values obtained for the optical parameters. Since the gels are primarily water, the refractive index was taken to be $n=1.33$, and the effective Fresnel reflectivity for the surface data was set at $R_{\text {eff }}=0.431$. At each value of the source-detector separation, phase and modulation data were fitted simultaneously with two fitting parameters: (1) absorption coefficient $\beta$ and (2) a parameter $f$, defined to be $f=r\left[(3 / 2) \sigma_{\mathrm{tr}}\right]^{1 / 2}$ for infinite-medium data and $f=\rho\left[(3 / 2) \sigma_{\mathrm{tr}}\right]^{1 / 2}$ for surface data. In infinite-medium equations (2.2.6) and (2.2.7), the transport scattering co- 
efficient $\sigma_{\text {tr }}$ and the fiber separation $r$ occur only in the combination defined in the parameter $f$. This is only approximately true in the semi-infinite-medium equations, but we continue to use these fitting parameters for reasons that will become clear in the discussion below.

The fitted values for absorption coefficient $\beta$ are plotted in Fig. 7 as a function of fiber separation. Three features of Fig. 7 deserve emphasis. First, fitting infinite-medium data with infinite-medium theory yields essentially the same values for $\beta$ as fitting surface data with semi-infinite-medium theory. The partial-current or the simplified extrapolated boundary condition seems to account successfully for the presence of the interface. Second, fitting surface data with infinite-medium theory yields values for $\beta$ that are too large by roughly $75 \%$. It is clearly important to use some boundary con- dition when one is analyzing surface data. Third, there is a tendency to overestimate $\beta$ at small fiber separations. In comparing Monte Carlo simulations with diffusion-theory predictions of diffuse reflectance versus fiber separation, we have noticed that diffusion theory deviates at values of $\rho$ less than $\sim 70 \%$ of attenuation length $\delta$ (see also Ref. 28). For the gel under consideration $\left(\beta=0.02 / \mathrm{cm}, \sigma_{\mathrm{tr}}=6 / \mathrm{cm}\right)$, that distance is $1.2 \mathrm{~cm}$. Indeed, the sharpest overestimates in Fig. 7 occur at values of $\rho$ less than $1.2 \mathrm{~cm}$ and may reflect a failure of diffusion theory. There is still a slight $\rho$ dependence at longer distances, but most of our FDPM data do not show this longer $\rho$ trend.

The fitted values for the parameter $f$ are plotted in Fig. 8 as a function of fiber separation. The dependence is linear as expected, and the surface data superpose

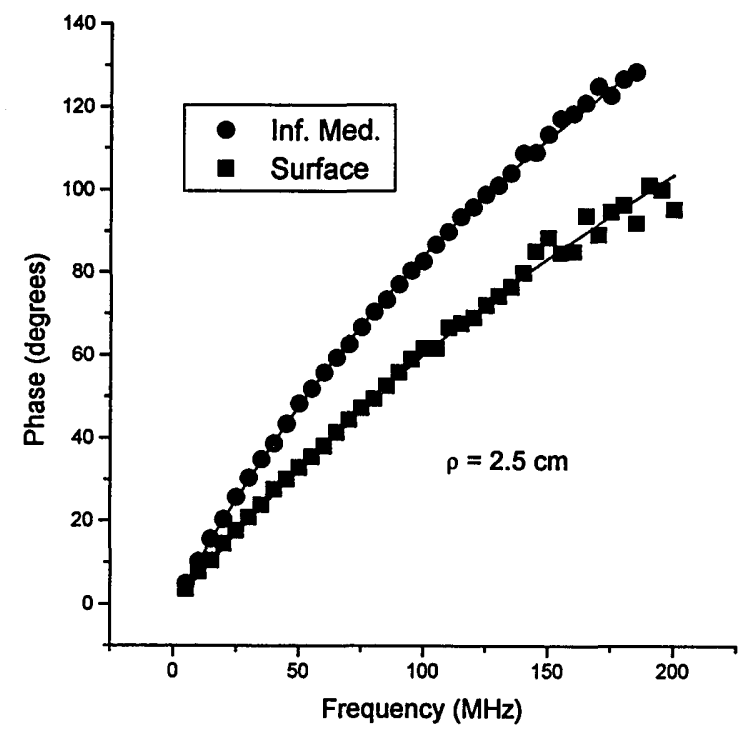

A

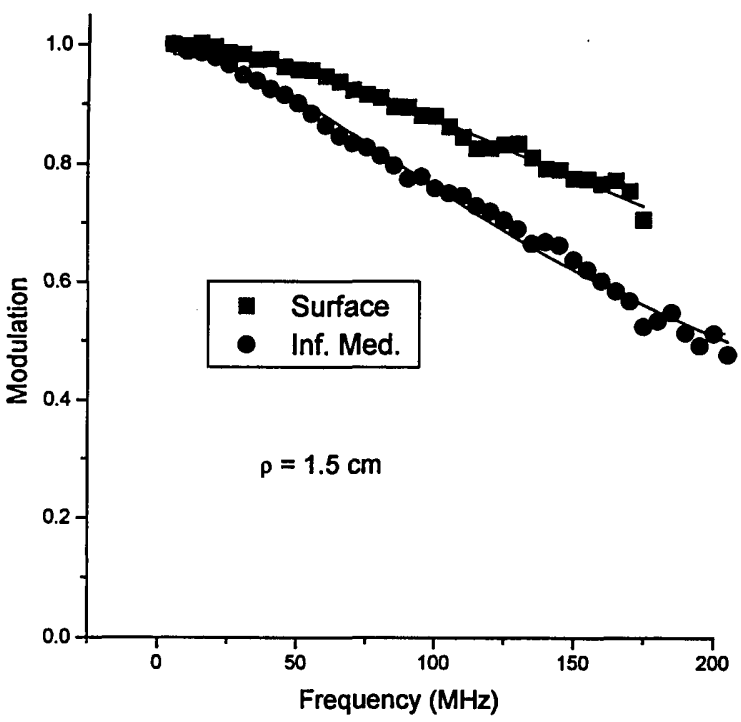

B

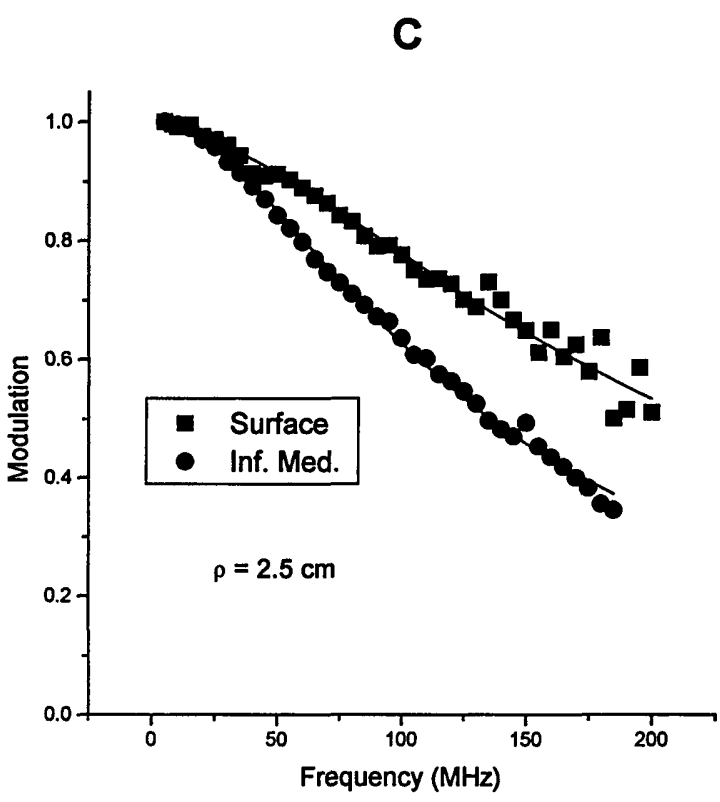

D

Fig. 6. FDPM phase (A, C) and modulation (B, D) data for an infinite medium (circles) and for a semi-infinite medium (squares). The tissue phantom was a $1 \%$ agar gel with $40 \%$ milk. The source-detector separation was $1.5 \mathrm{~cm}$ for the data in A and B and $2.5 \mathrm{~cm}$ for the data in $\mathrm{C}$ and $\mathrm{D}$. The solid curves represent nonlinear least-squares fits to Eqs. (2.2.6) and (2.2.7) for the infinite-medium data and to Eqs. (2.4.7) and (2.4.8) or (2.7.1) and (2.7.2) for the surface data. The fits were performed with $n=1.33$ and $R_{\text {eff }}=0.431$. 


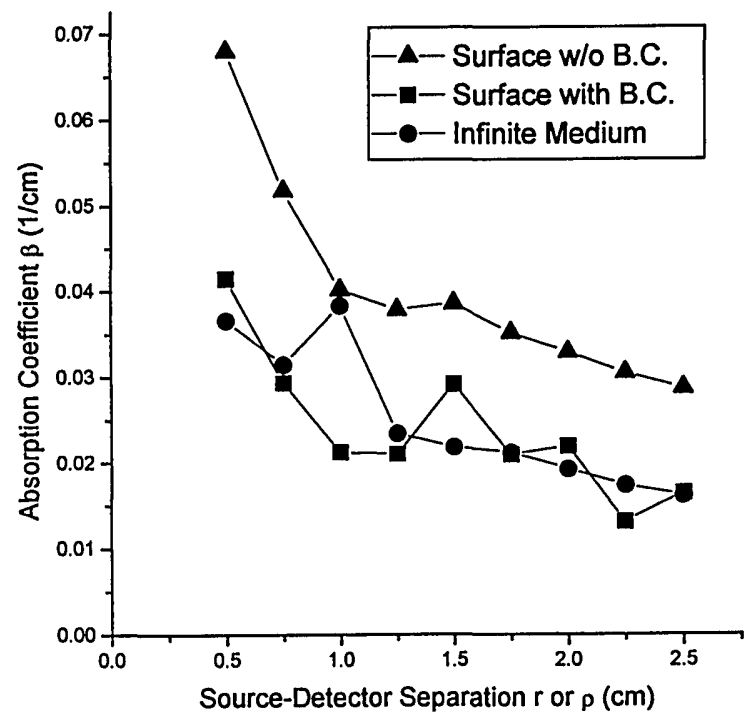

Fig. 7. Values for absorption coefficient $\beta$ derived from fits to phase and modulation data at nine different values of the source-detector separation. The tissue phantom was a $1 \%$ agar gel with $40 \%$ milk (as in Fig. 6). The circles are fits to infinite-medium data with Eqs. (2.2.6) and (2.2.7), and the squares are fits to surface data with Eqs. (2.4.7) and (2.4.8) or (2.7.1) and (2.7.2). The triangles are fits to surface data with infinite-medium equations (2.2.6) and (2.2.7), i.e., with no account taken of the presence of the boundary. B.C., boundary condition.

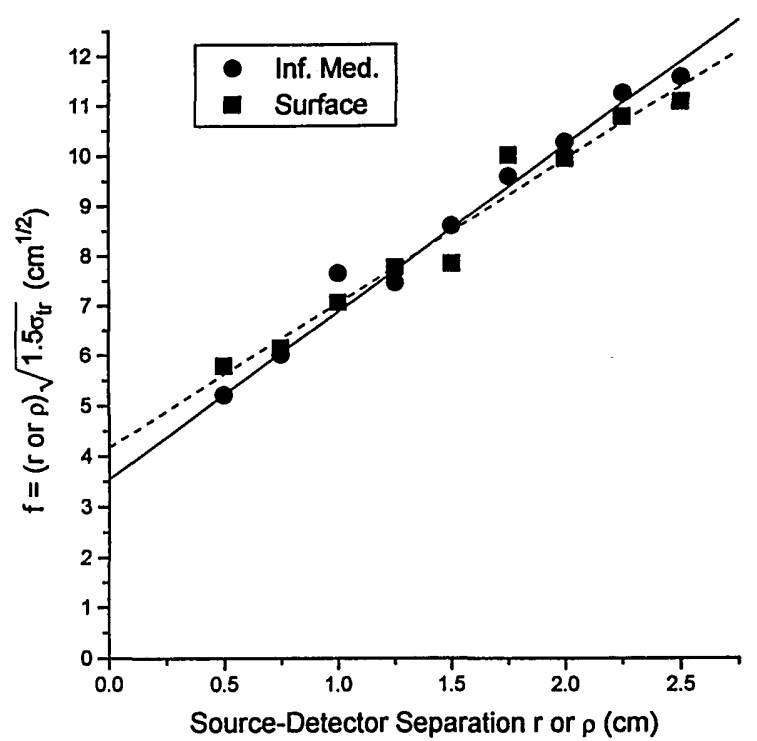

Fig. 8. Values for the parameter $f$ derived from fits to phase and modulation data at nine different values of the sourcedetector separation. The tissue phantom was a $1 \%$ agar gel with $40 \%$ milk (as in Figs. 6 and 7). The circles are fits to infinite-medium data with Eqs. (2.2.6) and (2.2.7), and the squares are fits to surface data with Eqs. (2.4.7) and (2.4.8) or (2.7.1) and (2.7.2). The curves represent linear least-squares fits to the infinite-medium $f$ values (solid lines) (with $r>1.0 \mathrm{~cm}$ ) and to the surface $f$ values (dashed curves) (with $\rho>1.0 \mathrm{~cm}$ ). From the slopes of the fits we find that $\sigma_{\mathrm{tr}}=7.6 \pm 1.8 / \mathrm{cm}$ from the infinite-medium data and $\sigma_{\mathrm{tr}}=6.4 \pm 0.5 / \mathrm{cm}$ from the surface data.

nicely on the infinite-medium data. From the slopes of linear fits to the two sets of data (using only points with $r$ or $\rho>1.0 \mathrm{~cm}$ ), values for the transport scattering coeffi- cient can be found: $\sigma_{\mathrm{tr}}=7.6 \pm 1.8 / \mathrm{cm}$ from the infinitemedium data and $\sigma_{\mathrm{tr}}=6.4 \pm 0.5 / \mathrm{cm}$ from the surface data. Again to demonstrate the importance of applying a boundary condition in the analysis of surface data, we obtained values for $f$ by fitting surface data with infinitemedium theory. A linear fit of these values (again for $\rho>1.0 \mathrm{~cm}$ ) yields $\sigma_{\mathrm{tr}}=3.4 \pm 1.0 / \mathrm{cm}$, an underestimate as predicted theoretically in Subsection 2.G.

A surprising feature of Fig. 8 is the nonzero intercept of the linear dependence of $f$ versus fiber separation. By definition, the $f$ data should extrapolate through the origin. This peculiar result can be traced to a nonzero intercept in a plot of the phase data versus fiber sepa-

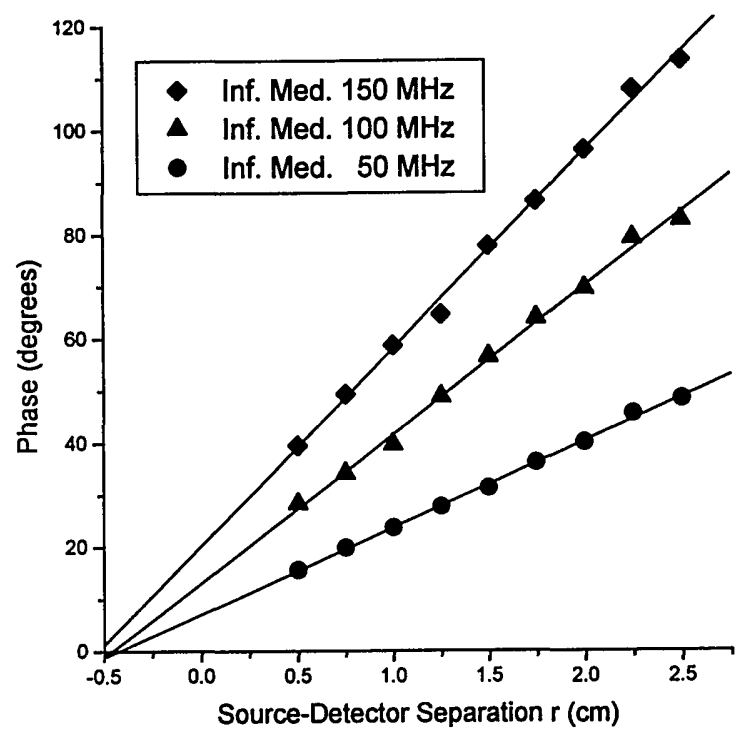

A

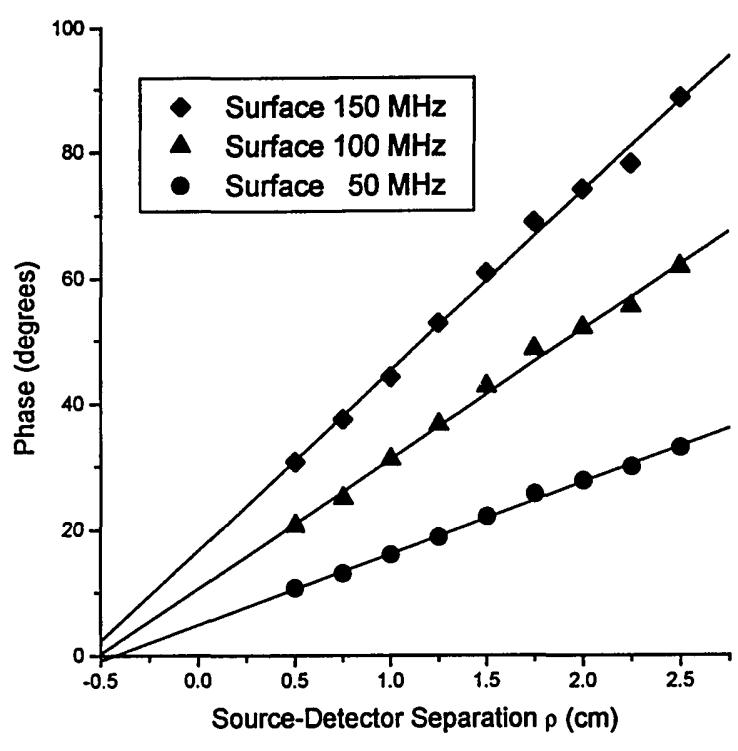

B

Fig. 9. Phase versus fiber separation for three different modulation frequencies: 50,100 , and $150 \mathrm{MHz}$. The source and the detector fibers were either pushed deep into the gel to simulate an infinite-medium geometry (A) or placed on the surface of the gel (B). The gel was the same one used in Figs. 6-8. For both infinite-medium and surface data the $x$ intercepts are approximately $-0.5 \mathrm{~cm}$. 


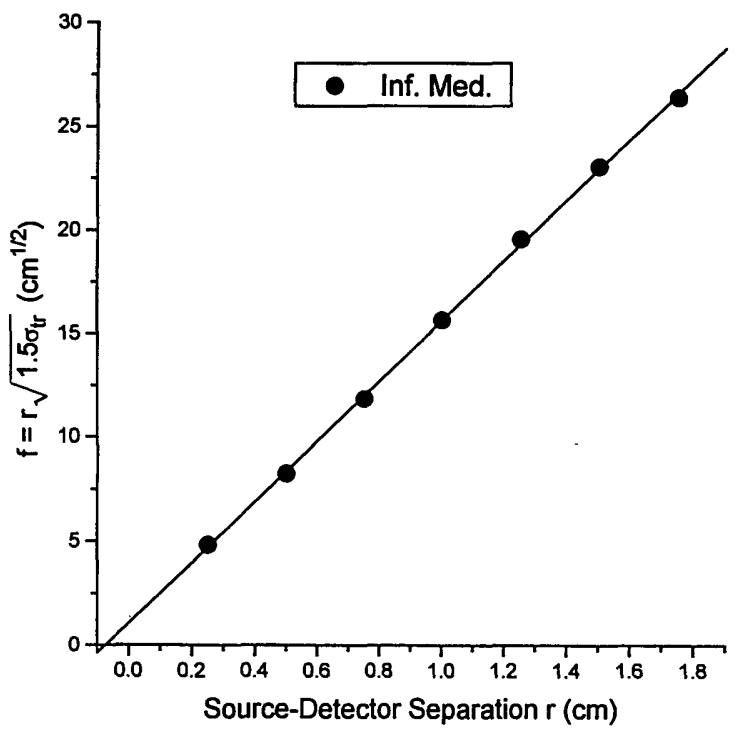

Fig. 10. Fitted values for parameter $f$ versus source-detector separation. The source and detector fibers were facing each other in an effectively infinite medium of $10 \%$ Intralipid. These FDPM measurements were made with just the argon-pump laser $(514 \mathrm{~nm})$ and have been described in more detail by Tromberg et $a l .{ }^{27}$ From the slope of the fitted line (solid line), the transport scattering coefficient is found to be $\sigma_{\mathrm{tr}}=140 / \mathrm{cm}$. The fitted value of the absorption coefficient is $\beta=0.021 / \mathrm{cm}$ (at $514 \mathrm{~nm}$ ). The $x$ intercept of the $f$-versus- $r$ line is $-0.07 \mathrm{~cm}$, considerably less than that of Fig. 8.

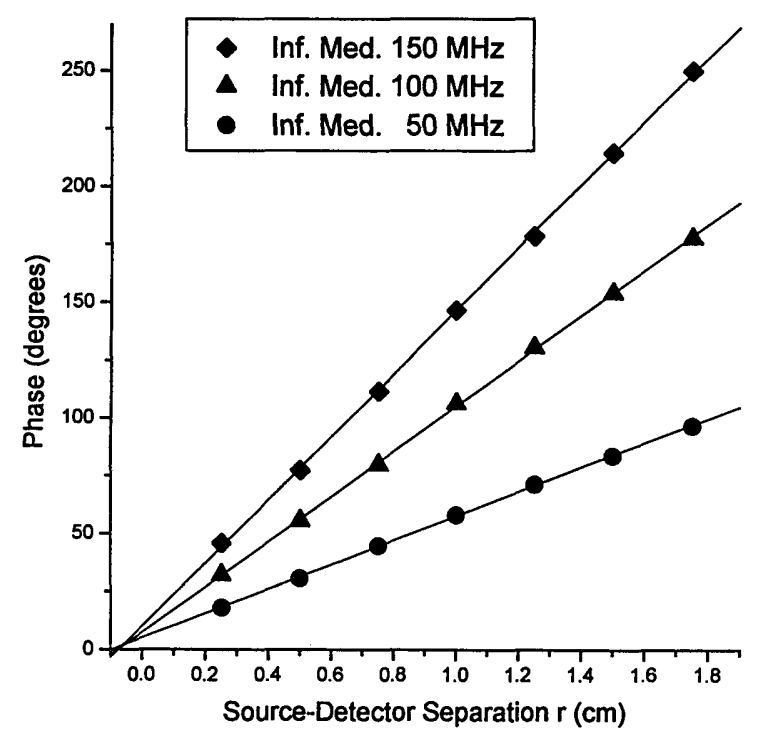

Fig. 11. Phase-versus-fiber $(r)$ separation for three different modulation frequencies: 50,100 , and $150 \mathrm{MHz}$. The source and detector fibers were facing each other in an effectively infinite medium of $10 \%$ Intralipid (as in Fig. 10). The $x$ intercepts of the fitted lines are approximately $-0.08 \mathrm{~cm}$, considerably less than those of Fig. 9.

ration (Fig. 9). Both infinite-medium and surface-data plots have an $x$ intercept of approximately $-0.5 \mathrm{~cm}$; i.e., the source seems to be $5 \mathrm{~mm}$ farther from the detector than the fiber separation would indicate. In retrospect, our model for the source of photon-density waves places the source at a distance $l_{\mathrm{tr}} \approx 1.7 \mathrm{~mm}$ beyond the end of the source fiber. This additional $1.7 \mathrm{~mm}$ from fiber tip to source plus the extra distance from source to detector fiber could cause the effective source-detector separation to be as much as $2 \mathrm{~mm}$ greater than the fiber separation (see Fig. 1). However, we are still puzzled by the additional factor of 2.5 needed to reach the $5-\mathrm{mm}$ $x$-intercept value. An interesting comparison is provided by FDPM data taken from a more strongly scattering sample $\left(l_{\mathrm{tr}}=0.0071 \mathrm{~cm}, \sigma_{\mathrm{tr}}=1 / l_{\mathrm{tr}}=140 / \mathrm{cm}\right)$ and with source and detector fibers facing each other. Figures 10 and 11 show the drastic reduction in intercepts both

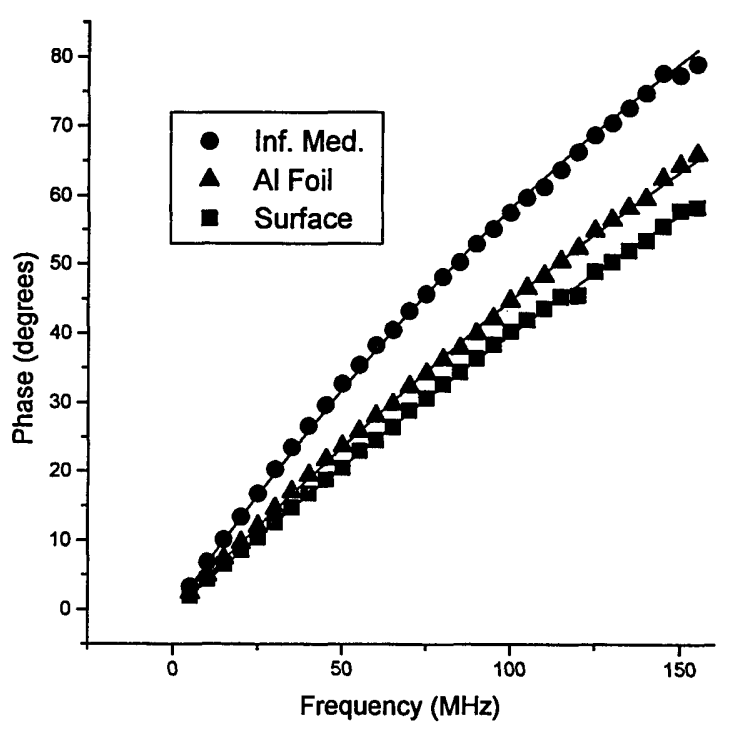

A

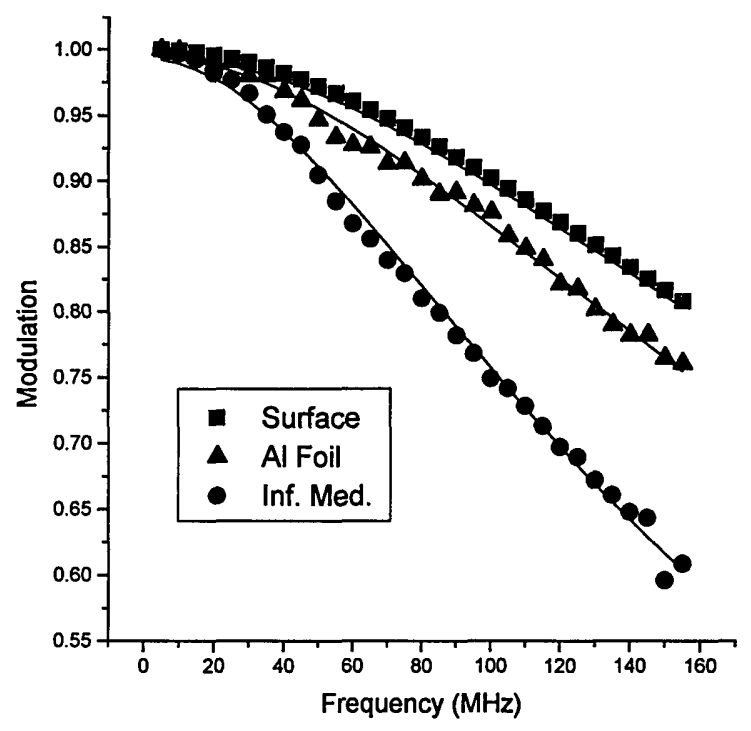

B

Fig. 12. Phase (A) and modulation (B) data collected under three sets of conditions: infinite-medium geometry (circles), semi-infinite-medium geometry (squares), and semi-infinitemedium geometry with the surface of the gel covered by aluminum foil (triangles). The gel was as for Fig. 6 (40\% milk), and the source-detector separation was $1.5 \mathrm{~cm}$. The solid curves are fits to Eqs. (2.2.6) and (2.2.7) for the infinite-medium data, to Eqs. (2.7.1) and (2.7.2) with $R_{\text {eff }}=0.431$ for the semi-infinite data, and to Eqs. (2.7.1) and (2.7.2) with $R_{\text {eff }}=0.8$ for the aluminum foil data. 


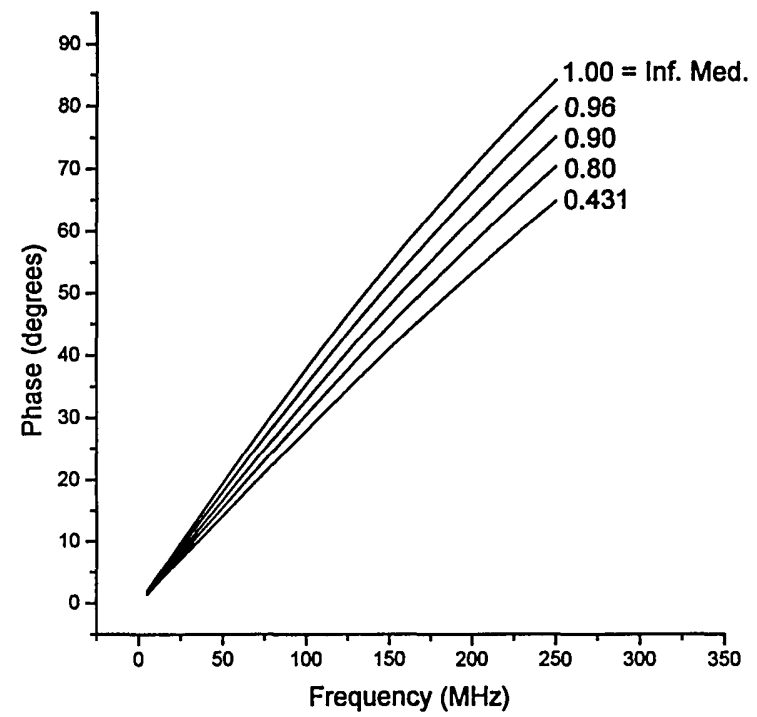

Fig. 13. Simulated phase data for an infinite medium (top curve) and for a semi-infinite medium with four different surface reflectivities: $R_{\text {eff }}=0.96,0.90,0.80,0.431$. The absorption coefficient is $\beta=0.05 / \mathrm{cm}$, and the transport scattering coefficient is $\sigma_{\mathrm{tr}}=10 / \mathrm{cm}$. The refractive index of the medium is $n=1.33$ for all cases, and the source-detector separation is $2.0 \mathrm{~cm}$.

in the $f$-versus- $r$ plot ( $x$ intercept $=-0.7 \mathrm{~mm}$ ) and in the phase-versus- $r$ plot $(x$ intercept $=-0.8 \mathrm{~mm})$. There are two reasons that these intercepts are smaller: first, the additional distance to the source point is now only $l_{\text {tr }}=0.07 \mathrm{~mm}$ and second, the additional distance to the source point may be compensated by the shorter distance to the detector fiber (the fibers are facing each other). We continue to explore this phenomenon and recommend the use of several source-detector separations when one is attempting to measure the transport scattering coefficient $\sigma_{\text {tr }}$.

We end this section by examining the effect of surface reflectivity $\left(R_{\text {eff }}\right)$ on phase and modulation. FDPM data were taken from a gel ( $40 \%$ milk) under three sets of conditions: (1) infinite-medium geometry, (2) semi-infinitemedium geometry with the source and detector fibers at the surface, and (3) semi-infinite-medium geometry as in condition (2) but with the surface of the gel covered with aluminum foil $(0.6-\mathrm{mm}$-diameter holes were cut for the fibers). Representative phase and modulation data are presented in Fig. 12. Note that the increase in $R_{\text {eff }}$ provided by the aluminum foil raises the phase data and reduces the modulation data.

According to the discussion in Subsection 2.D, an increase in $R_{\text {eff }}$ toward a value of 1.0 yields phase and modulation data closer to infinite-medium values. Figure 13 illustrates just how close $R_{\text {eff }}$ must be to 1.0 for the phase and modulation data to approach the infinite-medium values. We found that the aluminum foil data in Fig. 12 were best fitted with a value for $R_{\text {eff }}$ of 0.8 . Interestingly, this value is identical to our foil reflectivity measurements performed at $650 \mathrm{~nm}$ with an integrating sphere. Although a highly reflective surface placed on biological tissue could in principle eliminate the effect of the boundary, Fig. 13 clearly indicates that the reflectivity would have to reach an impractical value of 0.98 to mimic an infinite-medium geometry reliably.

\section{ACKNOWLEDGMENTS}

This research was performed with the support of grant WF 16493 from the Whitaker Foundation, grant R29GM-50958 from the National Institutes of Health, and Beckman Instruments, Inc. In addition, we acknowledge program support to the Beckman Laser Institute and Medical Clinic through grant DE-FG03-91ER61227 from the U.S. Department of Energy, grant 5P41-RR01192 from the National Institutes of Health, and grant N0001491-C-0134 from the U.S. Office of Naval Research. T.-T. Tsay was supported by the Hewitt Foundation, M. S. McAdams by the Society for Lasers in Medicine and Surgery, and T.-C. Feng by the Howard Hughes Medical Institute. The authors thank S. J. Madsen for a critical review of the manuscript and $\mathrm{K}$. Vu and E. Cho for preparation of the tissue phantoms.

Address any correspondence to Bruce J. Tromberg, Beckman Laser Institute and Medical Clinic, University of California, Irvine, Irvine, California 92715.

\section{REFERENCES AND NOTES}

1. See, for example, the three special journal issues on biomedical optics: Appl. Opt. 28(12), (1989); Appl. Opt. 32(4), (1993); Opt. Eng. 32(2), (1993).

2. B. Chance, J. Leigh, H. Miyake, D. Smith, S. Nioka, R. Greenfield, M. Finlander, K. Kaufmann, W. Levy, M. Yound, P. Cohen, H. Yodshioka, and R. Boretsky, "Comparison of time-resolved and unresolved measurements of deoxyhemoglobin in brain," Proc. Natl. Acad. Sci. (USA) 85, 4971-4975 (1988).

3. A. Knuttel, J. M. Schmitt, and J. R. Knutson, "Spatial localization of absorbing bodies by interfering diffusive photondensity waves," Appl. Opt. 32, 381-389 (1993).

4. D. A. Boas, M. A. O'Leary, B. Chance, and A. G. Yodh, "Scattering of diffuse photon density waves by spherical inhomogeneities within turbid media: analytic solution and applications," Proc. Natl. Acad. Sci. (USA) 91, 4887-4891 (1994).

5. A. Ishimaru, "Diffusion of light in turbid material," Appl. Opt. 28, 2210-2215 (1989).

6. J.D. Moulton, "Diffusion modelling of picosecond laser pulse propagation in turbid media," master's dissertation (McMaster University, Hamilton, Ont., 1990).

7. M. S. Patterson, S. J. Madsen, J. D. Moulton, and B. C. Wilson, "Diffusion representation of photon migration in tissue," in IEEE Microwave Theory and Techniques Symposium Digest, Vol. BB-1 (Institute of Electrical and Electronics Engineers, New York, 1991), pp. 905-908.

8. T. J. Farrell, M. S. Patterson, and B. Wilson, "A diffusion theory model of spatially resolved, steady-state diffuse reflectance for the noninvasive determination of tissue optical properties in vivo," Med. Phys. 19, 879-888 (1992).

9. R. Aronson, "Extrapolation distance for diffusion of light," in Photon Migration and Imaging in Random Media and Tissues, B. Chance and R. Alfano, eds., Proc. Soc. Photo-Opt. Instrum. Eng. 1888, 297-305 (1993).

10. M. Keijzer, W. M. Star, and P. R. M. Storchi, "Optical diffusion in layered media," Appl. Opt. 27, 1820-1824 (1988).

11. J. X. Zhu, D. J. Pine, and D. A. Weitz, "Internal reflection of diffusive light in random media," Phys. Rev. A 44, 3948-3959 (1991).

12. A. Lagendijk, R. Vreeker, and P. DeVries, "Influence of internal reflection on diffusive transport in strongly scattering media," Phys. Lett. A 136, 81-88 (1989).

13. K. M. Case and P. F. Zweifel, Linear Transport Theory (Addison-Wesley, Reading, Mass., 1967), Sec. 1.3.

14. J. J. Duderstadt and L. J. Hamilton, Nuclear Reactor Analysis (Wiley, New York, 1976).

15. B. Davison, Neutron Transport Theory (Oxford, London, 1958). 
16. S. Glasstone and M. C. Edlund, The Elements of Nuclear Reactor Theory (Van Nostrand, Princeton, N.J., 1952), Chaps. 5 and 14.

17. F. P. Bolin, L. E. Preuss, R. C. Taylor, and R. J. Ference, "Refractive index of some mammalian tissues using a fiber optic cladding method," Appl. Opt. 28, 2297-2302 (1989).

18. L. O. Svasaand, B. J. Tromberg, R. C. Haskell, T.-T. Tsay, and M. W. Berns, "Tissue characterization and imaging using photon density waves," Opt. Eng. 32, 258-266 (1993).

19. R. Graaff, A. C. M. Dassel, M. H. Koelink, F. F. M. de Mul, J. G Aarnoudse, and W. G. Zijlstra, "Optical properties of human dermis in vitro and in vivo," Appl. Opt. 32, 435-447 (1993).

20. J. B. Fishkin and E. Gratton, "Propagation of photon-density waves in strongly scattering media containing an absorbing semi-infinite plane bounded by a straight edge," J. Opt. Soc. Am. A 10, 127-140 (1993).

21. M. S. Patterson, B. Chance, and B. C. Wilson, "Time resolved reflectance and transmittance for the non-invasive measurement of tissue optical properties," Appl Opt. 28, 2331-2336 (1989).
22. H. S. Carslaw and J. C. Jaeger, Conduction of Heat in Solids (Oxford, London, 1959).

23. G. H. Bryan, "An application of the method of images to the conduction of heat," Proc. London Math. Soc. 22, 424-430 (1891).

24. S. J. Madsen, B. C. Wilson, M. S. Patterson, Y. D. Park, S. L. Jacques, and Y. Hefetz, "Experimental tests of a simple diffusion model for the estimation of scattering and absorption coefficients of turbid media from time-resolved diffuse reflectance measurements," Appl. Opt. 31, 3509-3517 (1992).

25. D. Ben-Avraham, H. Taitelbaum, and G. H. Weiss, "Boundary conditions for a model of photon migration in a turbid medium," Lasers Life Sci. 4, 29-36 (1991).

26. V. Allen and A. L. McKenzie, "The modified diffusion dipole model," Phys. Med. Biol. 36, 1621-1638 (1991).

27. B. J. Tromberg, L. O. Svaasand, T.-T. Tsay, and R. C. Haskell, "Properties of photon density waves in multiplescattering media," Appl. Opt. 32, 607-616 (1993).

28. P. D. Kaplan, M. H. Kao, A. G. Yodh, and D. J. Pine, "Geometric constraints for the design of diffusing-wave spectroscopy experiments," Appl. Opt. 32, 3828-3836 (1993). 